\title{
The Role of Gravitational Instabilities in the Feeding of Supermassive Black Holes
}

\author{
Giuseppe Lodato \\ Dipartimento di Fisica, Università degli Studi di Milano, Via Celoria 16, Milano, Italy \\ Correspondence should be addressed to Giuseppe Lodato, giuseppe.lodato@unimi.it
}

Received 28 August 2011; Accepted 7 October 2011

Academic Editor: Francesco Shankar

Copyright () 2012 Giuseppe Lodato. This is an open access article distributed under the Creative Commons Attribution License, which permits unrestricted use, distribution, and reproduction in any medium, provided the original work is properly cited.

I review the recent progresses that have been obtained, especially through the use of high-resolution numerical simulations, on the dynamics of self-gravitating accretion discs. A coherent picture is emerging, where the disc dynamics is controlled by a small number of parameters that determine whether the disc is stable or unstable, whether the instability saturates in a self-regulated state or runs away into fragmentation, and whether the dynamics is local or global. I then apply these concepts to the case of AGN discs, discussing the implications of such evolution on the feeding of supermassive black holes. Nonfragmenting, self-gravitating discs appear to play a fundamental role in the process of formation of massive black hole seeds at high redshift $(z \sim 10-15)$ through direct gas collapse. On the other hand, the different cooling properties of the interstellar gas at low redshifts determine a radically different behaviour for the outskirts of the accretion discs feeding typical AGNs. Here the situation is much less clear from a theoretical point of view, and while several observational clues point to the important role of massive discs at a distance of roughly a parsec from their central black hole, their dynamics is still under debate.

\section{Introduction}

The accretion discs surrounding the growing supermassive black holes $(\mathrm{SMBH})$ in active galactic nuclei (AGN) are expected to become gravitationally unstable at a distance of $\sim 0.01 \mathrm{pc}$ from the black hole $[1,2]$. Traditionally, this occurrence has been interpreted in relation to star formation: a self-gravitating disc, in this picture, would rapidly fragment and form stars $[3,4]$. At the same time, it has been noted very early that the development of gravitational instability may also act as an efficient mechanism to produce torques through the effect of the resulting spiral structure and thus might be very effective in redistributing angular momentum within the disc and promote accretion [5-7]. As we shall see, the modern debate about these issues still concentrates on these two extreme cases. While we now have a much clearer understanding of the mechanism of growth and saturation of the instability in gaseous discs, and-especially though the use of high-resolution numerical simulationswe have clarified what are the main parameters regulating the disc structure and evolution, some questions are still unanswered. Are massive discs effectively truncated by star formation at the radius where they become self-gravitating, thus preventing accretion beyond these scales? Or does accretion proceed effectively through gravitational torques even in fragmenting discs, allowing the central black holes to be fed by gas on parsec scales?

From the observational point of view, on the one hand, it has now become quite clear that fragmentation in massive discs can be very important for the formation of compact, young stellar clusters in AGNs, and in particular in our own Galaxy $[8,9]$. On the other hand, it is also clear that rotating gaseous discs exist on parsec scales in AGN [10-12], often displaying a clear Keplerian rotation [10].

All the issues discussed above bear important consequences not just for the dynamics of the disc itself, but, in a broader context, relate to the overall process of coevolution between the supermassive black hole and the host galaxy. The efficiency of star formation in the disc, the efficiency of the accretion process, and the related timescales and duty cycle of AGN activity are all often assumed as subgrid physics in simulations of galactic evolution on larger scales [17-19], which turn out to be quite sensitive to the chosen subgrid prescriptions. 
In this contribution, I will not try to give an exhaustive answer to the questions above. I will rather give an account of the progresses we have made in recent years in our understanding of these phenomena and highlight their importance in several contexts related to the feeding of supermassive black holes. I will first summarize, in Section 2, the state of the art about the evolution of gravitational instabilities in gaseous discs, from a purely theoretical point of view. In Section 3, I will describe the possible importance of gravitational torques in the formation of the seeds of supermassive black holes by direct collapse in the early evolution of pregalactic discs. In Section 4, I will address the issue of feeding the $\mathrm{SMBH}$ in AGN through gravitational torques, and the related issue of fragmentation of AGN discs. Finally, in Section 5 I will draw some conclusions.

\section{Gravitational Instabilities in Gaseous Discs}

The issue of the nonlinear evolution of gravitational instabilities in gaseous discs has been studied in great detail over the last $10-15$ years $[13,20-25]$. As a result, despite the differences in the numerical methods adopted and in the setup used, a coherent picture of the overall dynamics is emerging. This issue has also been covered in several reviews, see for example Lodato [26] and Durisen et al. [27], and the reader is referred to these papers for further details and for an application of these concepts to different astrophysical systems (such as protostellar and protoplanetary discs), which share similar characteristics.

Consider an accretion disc with surface density $\Sigma(R)$, where $R$ is the cylindrical distance to the central object of mass $M$, around which the disc is rotating in approximately centrifugal balance with angular velocity $\Omega(R)$. Let us also define the epicyclic frequency $\kappa$, which is equal to $\Omega$ in the case in which the rotation curve of the disc is Keplerian, $\Omega^{2}=G M / R^{3}$. If the disc mass is high enough $\left(M_{\text {disc }} \approx M\right)$, deviations from Keplerian rotation might arise [28] and $\kappa$ is not going to be exactly equal to $\Omega$. The disc midplane temperature is $T(R)$, and the sound speed is $c_{s} \propto T^{1 / 2}$. The disc thickness is $H=c_{s} / \Omega$ for a non-self-gravitating disc and $H=c_{s}^{2} / \pi G \Sigma$ for a self-gravitating disc: we shall see that for gravitationally unstable discs the two definitions are equivalent. For most cases, we will consider thin discs, for which $H / R \ll 1$.

Fundamentally, the dynamics of self-gravitating accretion discs depends on three dimensionless parameters. Firstly, there is the well-known axisymmetric stability parameter $Q=c_{s} \kappa / \pi G \Sigma$ [29]. The second important parameter is the ratio between the cooling time $t_{\text {cool }}$ and the dynamical time $t_{\text {dyn }}=\Omega^{-1}$, a parameter often called $\beta=\Omega t_{\text {cool }}$. Thirdly, we have the ratio between the disc mass and the central object mass $M_{\text {disc }} / M$. As we shall see, each of these parameters controls some important features about the evolution of the gravitational instability.

2.1. The Role of Q: Linear Stability. As mentioned above, the basic, and most widely used, criterion to determine the stability of a massive disc against gravitational perturbations is related to the linear dispersion relation in the WKB approximation for an infinitesimally thin disc [30]:

$$
(\omega-m \Omega)^{2}=c_{s}^{2} k^{2}-2 \pi G \Sigma|k|+\kappa^{2},
$$

where $\omega$ is the frequency of the perturbation, $k$ is the radial wave number, and $m$ is the azimuthal wave number. The above dispersion relation is quadratic in $k$ from which one easily sees that, for $m=0$ (axisymmetric perturbations), $\omega^{2}$ is positive (and the perturbation is stable) at all wavelengths if

$$
Q=\frac{c_{s} \kappa}{\pi G \Sigma}>1
$$

Marginal stability occurs at $Q=1$.

Here we should note that the above (local) dispersion relation is strictly speaking only appropriate for infinitesimally thin discs and for tightly wound perturbations $(m / k R \ll 1)$ for which the WKB approximation holds. Finite thickness effects generally act so as to dilute the effect of self-gravity, thus making the disc more stable and decreasing the marginal stability value of $Q$ below unity (i.e., allowing a colder disc to remain stable). On the contrary, global perturbations are more unstable [31] thus effectively increasing the marginal stability value of $Q$.

For most cases considered here, the disc is close to being in Keplerian rotation, for which $\kappa=\Omega$. In this case, it is easy to show that the requirement of marginal stability $(Q \approx 1)$ is equivalent to

$$
\frac{M_{\mathrm{disc}}}{M} \approx \frac{H}{R},
$$

where $M_{\mathrm{disc}}=\pi \Sigma R^{2}$ is a measure of the enclosed disc mass within radius $R$. Thus, for marginally stable discs, "thin" and "light" on the one hand and "thick" and "massive" on the other hand are equivalent. Also note that, as mentioned above, when $Q \approx 1$, the two expressions for the disc thickness in the non-self-gravitating and in the self-gravitating regime are indeed equivalent.

AGN discs are generally quite thin, with $H / R \approx 10^{-3}$, and thus even a relatively light disc, much less massive than the central black hole, can be marginally stable. It is then easy, based on standard models of accretion discs around supermassive black holes [32], to calculate the distance from the black hole at which the disc first becomes gravitationally unstable $[2,33]$. This turns out to be of the order of $10^{3} R$. (where $R_{\bullet}$ is the Schwarzschild radius of the black hole), or $0.01 \mathrm{pc}$, for a $10^{8} M_{\odot}$ black hole. Thus, discs that extend beyond this radius are going to be gravitationally unstable: in order to determine their evolution, we need to understand the behaviour of the instability at the nonlinear stage: this is addressed in the next subsection.

2.2. The Role of $\beta$ : Fragmentation versus Self-Regulation. The details of the nonlinear evolution of the gravitational instability are best understood through the use of hydrodynamical simulations, which include the disc self-gravity. However, before discussing such simulations, let us make 
some preliminary remarks to guide us in the interpretation of the results of the simulation.

The very same fact that the linear stability of the disc depends on $Q$, which is directly proportional to the sound speed $c_{s} \propto T^{1 / 2}$ (where $T$ is the disc temperature), offers a possible way to predict the nonlinear evolution of the system. In fact, the development of the instability will act as to feed back energy into the disc and to heat it up, thus making it more stable. In practice, the linear stability condition works as a "thermostat" for the disc, so that heating turns on only when $Q$ drops below the marginal stability value, which we have seen is of order unity. If the thermostat works, we would expect the disc to be always close to marginal stability, at least under some conditions, in a so-called "self-regulated state" $[34,35]$.

From a numerical point of view, it is clear then that if we want to catch the dynamics associated with self-regulation, we need to make sure that the instability is able to feed back energy into the disc, and we should not then use isothermal simulations (such as the pioneering ones of [36]), which by constraint do not allow the disc to heat up. At the same time, we need to make sure that the disc is able to cool; otherwise, once the instability sets in, it will stabilize the disc forever (cf. the "perennial heating" problem for the spiral structure in galaxies), and we should thus also avoid pure $\mathrm{N}$ body simulations, unless special arrangements are made to artificially cool the disc down [37].

One such approach has been taken by Gammie [20], who ran local, shearing-sheet simulations of razor-thin discs, which were allowed to heat up through shocks and $p \mathrm{~d} V$ work and to cool down, according to a simple cooling prescription, such that

$$
\frac{\mathrm{d} T}{\mathrm{~d} t}=-\frac{T}{t_{\text {cool }}},
$$

where the cooling time $t_{\text {cool }}$ is a free input parameter for the simulation. While more complex approaches, which consider the details of the radiative transfer within the disc [38], can certainly be adopted, such an approach should be considered as a useful "numerical experiment," in order to evaluate the disc response as a function of the main parameters, rather than as a "realistic" simulation of some particular system. Having clarified the main dependencies from the physical parameters, we may then establish the disc response in any particular system. Following this approach, a number of papers have considered the details of the process $[13,21,24,25]$, extending the simulations to full $3 \mathrm{D}$ and considering thus global and potentially thick configurations, as a function of the main parameters of the system, such as the disc mass and thickness. Here, I will present a summary of the main results concerning the issue of fragmentation and self-regulation of the instability. In the next subsection, I will address the important issue of the locality of the induced transport.

It turns out that the behaviour of the disc is actually determined by the ratio of $t_{\text {cool }}$ to the dynamical time in the disc,

$$
\beta=\Omega t_{\text {cool }}
$$

It should be noted that, in most of the simulations described here $[13,20,21,24,25]$, the parameter $\beta$ is taken to be a single-free parameter for each simulation, with no dependance on either time or position in the disc. This is certainly not realistic, as in fact the cooling time should and will depend on the local microphysics associated with the disc opacity and radiative properties. These simulations should thus be regarded as simple "numerical experiments," where we test the disc response in a controlled configuration, as a function of the main parameters. For an actual, astrophysically relevant disc, we would thus calculate at any given radius the cooling properties and thus infer the disc behaviour from our controlled experiments. In doing this, care should be taken that the results are not affected by global effects (see below) or by nonlinear effects induced by a temperature dependence of the cooling rate, which has been studied by Johnson and Gammie [39] and Cossins et al. [40].

If the cooling timescale is larger than a few dynamical timescales, an initially stable (large $Q$ ) disc cools down until $Q$ becomes of the order of unity. At this stage, the disc becomes gravitationally unstable and develops a spiral structure which provides a heating source, through compressional heating and shock dissipation, able to balance the externally imposed cooling. Once in thermal equilibrium, the disc is characterized by an approximately constant value of $Q$ very close to marginal stability. In such a state, a spiral structure persists in the disc, to provide the required heating. Therefore, the self-regulation mechanism described above determines the disc structure and evolution. Figure 1(a) shows the result of one such simulations, where in this case $\beta=10$ and the total disc mass $M_{\text {disc }}=0.1 M$ [13]. The colour plot shows the disc surface density, in which a spiral structure is clearly seen. Figure 1(b) shows the azimuthally and vertically averaged value of $Q$ as a function of radius, for several simulations with the same mass ratio but with different values of $\beta$, as indicated. The disc in this case extends from $R=0.25$ to $R=25$ in code units. It is then seen that far from the boundaries (where the density drops and $Q$ correspondingly grows) the disc is self-regulated, with $Q \approx 1$ over a wide radial range. Cossins et al. [13] have also computed the amplitude of the perturbed surface density as a function of $\beta$. Analysis of the disc structure showed that while the cooling rate $\beta$ does not influence the spectrum of wavenumbers that are excited, it does affect that amplitude of the density perturbations, such that

$$
\frac{\delta \Sigma}{\Sigma} \approx \frac{1}{\sqrt{\beta}}
$$

which is shown in Figure 2. Thus as the cooling becomes more rapid (and thus as $\beta$ decreases), the amplitude of the density perturbation increases. Similarly, it was found [13] that the spectrum of the radial wavenumber $k$ peaks strongly where $k H=1$, a result that can be predicted from the dispersion relation (1) but has now also been demonstrated numerically. This result is independent of both the cooling rate and the disc to star mass ratio.

The behaviour described above changes when the cooling time is decreased to smaller values [20]. In this case, 


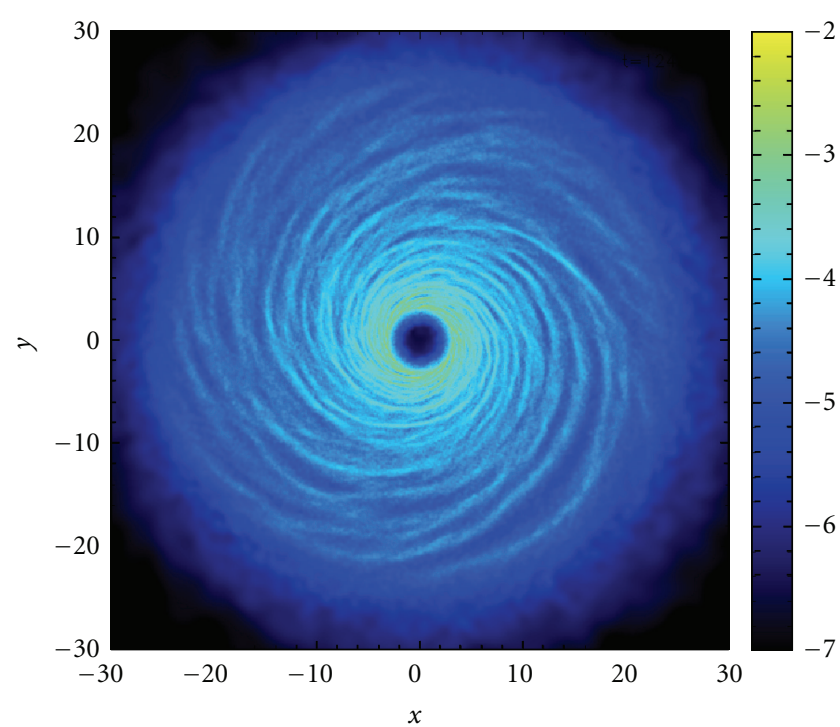

(a)

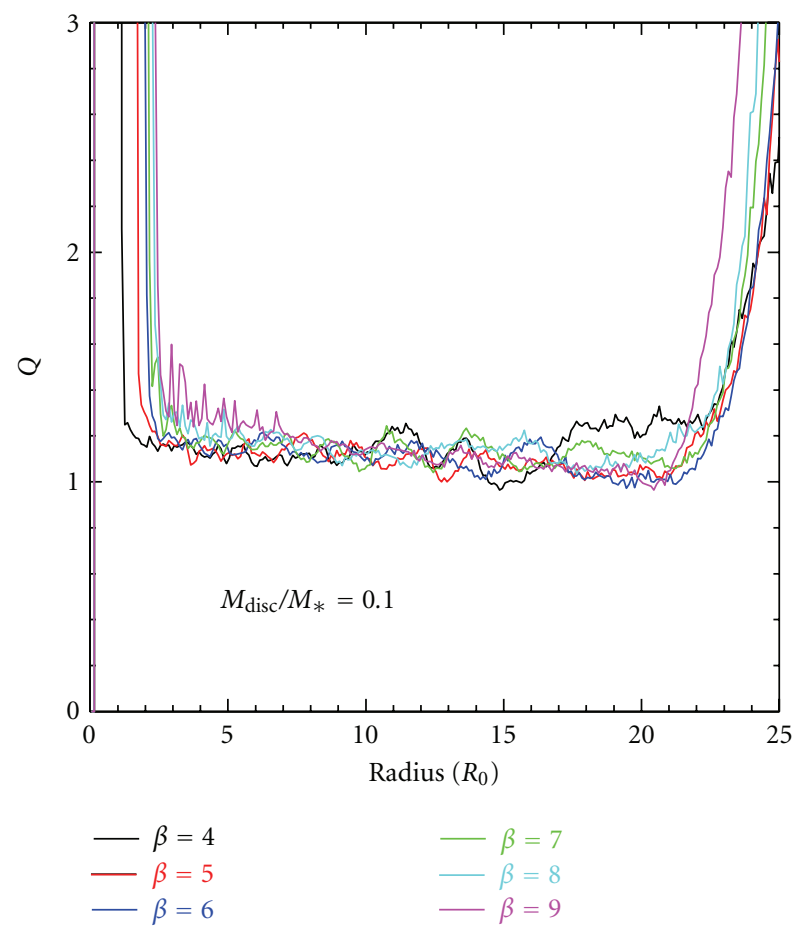

(b)

Figure 1: (a) Surface density of a self-gravitating disc, with $M_{\text {disc }}=$ $0.1 M$ and with $\beta=10$, where a tightly wound spiral structure is clearly seen. (b) Azimuthally averaged profiles of $Q$ as a function of radius, for several simulations, with varying $\beta$, as indicated. From Cossins et al. [13].

the disc does not reach a quasisteady self-regulated state but rather fragments into several bound objects. Figure 3 show the results of a simulation very similar to the one displayed in Figure 1, but where the cooling time is decreased to $\beta=3$ [24]. The presence of numerous high-density clumps is clearly seen. This result can be understood in

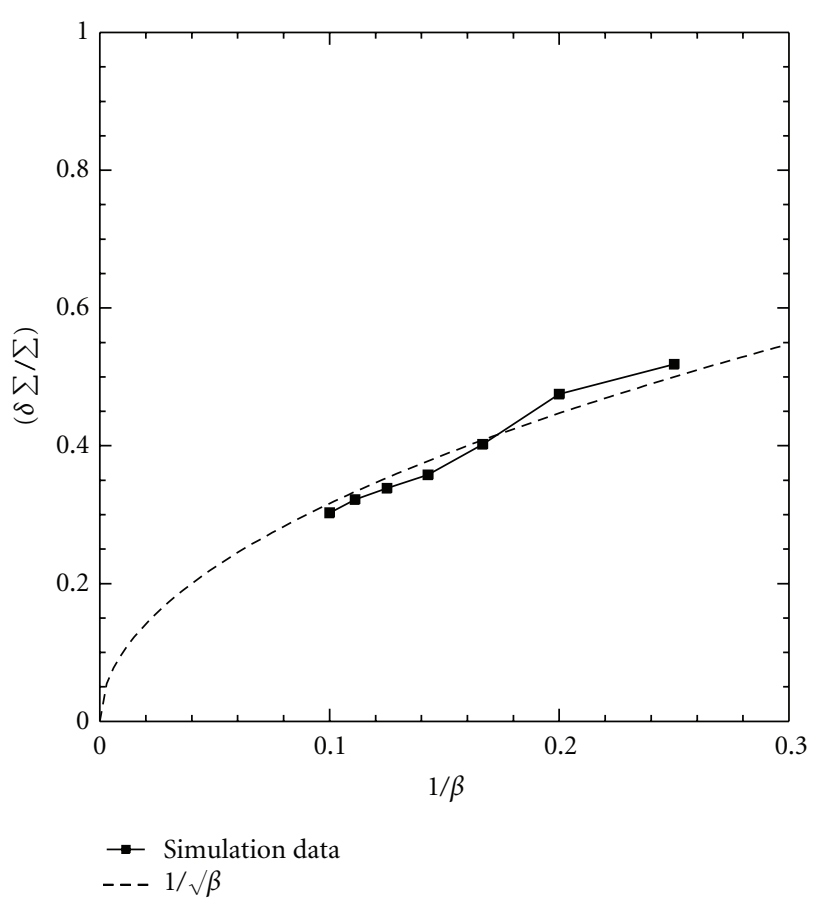

FIGURE 2: Variation of the radially and azimuthally averaged relative surface density perturbation amplitude $\delta \Sigma / \Sigma$ with the inverse cooling parameter $1 / \beta$. From Cossins et al. [13].

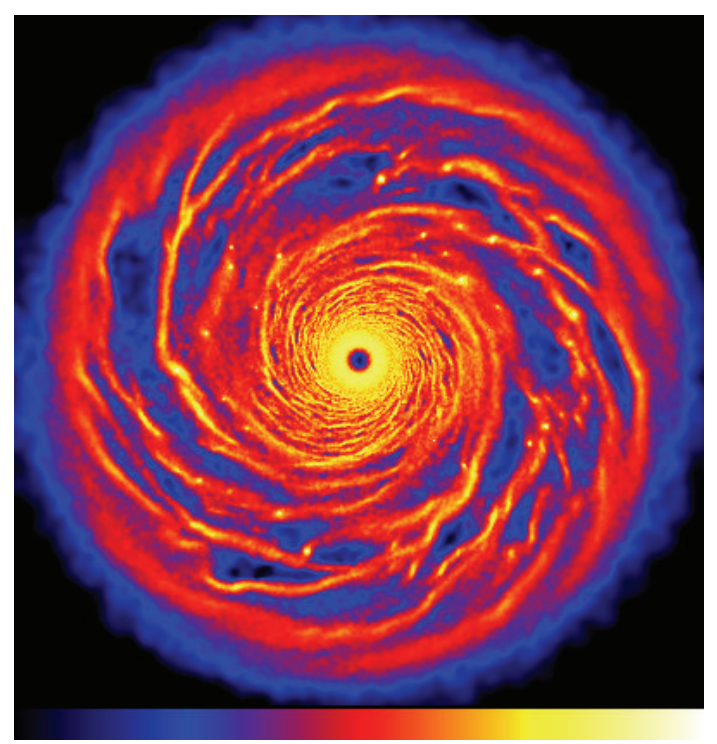

FIgURE 3: Numerical simulation of a self-gravitating disc with $M_{\text {disc }}=0.1 M$ and $\beta=3$. Once unstable, the disc breaks up into numerous gravitationally bound clumps.

the following way, by adopting a local approach to describe the instability. In a gravitationally unstable disc, the typical growth timescale of unstable perturbations is of the order of the dynamical timescale $\Omega^{-1}$. The nonlinear stabilization of the perturbation only works if the heat generated by compression and shocks is not removed too efficiently from the disc through cooling. Since the perturbation grows on the 
dynamical timescale, if we want to avoid fragmentation, we require that cooling acts on a longer timescale. Note that the requirement that the cooling timescale be shorter than the dynamical timescale in order to result in fragmentation has been known for several years, even outside the context of disc instability [41, 42]. Note also that the existence of a critical cooling time below which the disc fragments can be easily related, through (6), to a maximum value of the perturbation amplitude that can be sustained by the disc.

The exact value of the threshold for fragmentation does depend somewhat on the specific numerical setup and ranges from $\beta=3$ to $\beta=6[20,24,43]$. Recently, the exact value of this threshold has been the subject of intense debate, as it was discovered [44] that the threshold value appeared to increase with increasing resolution in smoothed particle hydrodynamics (SPH) simulations (see also $[45,46]$ ). The same behaviour has also been seen in grid-based simulations [47], and it has been shown that it actually depends on the chosen initial conditions. When carefully chosen initial conditions are used, the threshold value for fragmentation does converge and it turns out to be indeed of the order of $\beta \approx 6$.

We can easily get a reasonable estimate of the mass of the fragments. Indeed, we expect $M_{\mathrm{frag}} \approx \pi \Sigma \lambda^{2}$, where $\Sigma$ is the local density and $\lambda \approx H$ is the typical wavelength associated with the instability. We thus obtain

$$
M_{\mathrm{frag}} \approx \pi \Sigma R^{2}\left(\frac{H}{R}\right)^{2} \approx\left(\frac{H}{R}\right)^{3} M,
$$

where in the last equality we have used the fact that, for a marginally stable disc, $\pi \Sigma R^{2} \approx(H / R) M$. For a typical AGN disc, where $H / R \approx 10^{-3}-10^{-2}$ and, say, $M \approx 10^{8} M_{\odot}$, the fragment mass thus corresponds to $0.1-100 M_{\odot}$.

2.3. The Role of $M_{\mathrm{disc}} / M$ : Global versus Local Dynamics. The issue of locality of the dynamics associated with gravitational instability is essential if one wants to construct simple viscous models for self-gravitating accretion discs [48, 49]. Indeed, it has been long realized that the spiral structure determined by the instability can efficiently transport angular momentum [50], and one may thus suppose that the instability, at the large scales where an AGN disc is unstable (and where probably the disc is too cold to support MHD instabilities, such as the magnetorotational instability, MRI), can produce the required "viscous" torque to allow the accretion of matter from $\sim$ parsec scales down to the innermost regions where the MRI takes over and releases the accretion fuel down to the SMBH.

In the standard $\alpha$-prescription for accretion disc viscosity [32], the relevant component of the viscous stress tensor $T_{R \phi}$ is simply parameterized in terms of the local pressure $P$, such that $T_{R \phi} \approx \alpha P$. The dimensionless parameter $\alpha$ is thus simply a measure of the stress tensor in units of the local pressure. One might thus be tempted to compute the stress tensor resulting from the spiral structure seen in the simulations described above and directly compute an equivalent $\alpha$ parameter associated to the instability. This would be obviously best done for the cases where the disc is self-regulated and the instability saturates at a given perturbation amplitude, as discussed above. However, a fundamental problem arises in this case. This is related to the fact that the gravitational instability, is an intrinsically longrange instability and it is not clear whether the transport of energy and angular momentum associated with it can be simply expressed in terms of a local viscous process [51].

The problem is best understood in terms of a WKB analysis of the energy and angular momentum fluxes associated with the instability [13]. For a local, viscous process the torque exerted on the disc $\dot{\mathscr{L}}_{\alpha}$ is related to the work done by viscosity $\dot{\varepsilon}_{\alpha}$ via the Keplerian rotation rate $\Omega$, such that

$$
\dot{\mathcal{E}}_{\alpha}=\Omega \dot{\mathcal{L}}_{\alpha}
$$

A similar but not equal relation governs the case where potentially global effects are mediated through wave transport. In a WKB analysis, the wave angular momentum and energy densities can be obtained [30], and in turn the waveinduced torque $\dot{\mathscr{L}}_{w}$ and power dissipation $\dot{\mathcal{E}}_{w}$ are found to be related via [13]

$$
\dot{\mathcal{E}}_{w}=\Omega_{p} \dot{\mathcal{L}}_{w}
$$

where the pattern speed of the spiral perturbation is given by $\Omega_{p}=\omega / \mathrm{m}$. The transport properties of gravitationally induced waves are therefore determined not by the rotation rate of the disc material (cf. (8)), but by the pattern speed of the density waves themselves. As these waves are excited or absorbed, the power exchanged with the background flow for a given stress is therefore significantly different than that dissipated by a viscous process that provides the same stress to the extent to which $\Omega_{p}$ is significantly different from $\Omega$. The relative level of global versus local transport can hence be quantified via the parameter $\xi$, where

$$
\xi=\left|\frac{\Omega-\Omega_{p}}{\Omega}\right| .
$$

The analysis of Cossins et al. [13] also allows a spectrally averaged pattern speed to be determined, and thus in turn the nonlocal transport fraction $\xi$ can be measured from the simulations. In agreement with Lodato and Rice [21, 25], this shows that transport by gravitational waves is a predominantly local process for the systems modeled, with $\xi \approx 10 \%$ for $M_{\mathrm{disc}} / M=0.1$ and increasing with increasing disc to star mass ratio. This is shown as a function of radius in the left-hand panels of Figure 4, where the increase in nonlocality is clearly seen with $q$. A corollary of this, seen from the form of (10), is that the waves remain on average close to corotation, $\Omega_{p} \approx \Omega$.

The right-hand panels of Figure 4 show a further interesting result obtained from the simulations of Cossins et al. [13] - the wave Mach numbers. While the heavy lines shows the values relative to an external inertial frame, the lighter lower lines give the Doppler-shifted Mach numbers $\mathcal{M}$, that is, those relative to a frame corotating with the flow. These Doppler-shifted values are almost exactly unity, implying that the density waves excited by the gravitational instability are only weakly supersonic, and furthermore this 

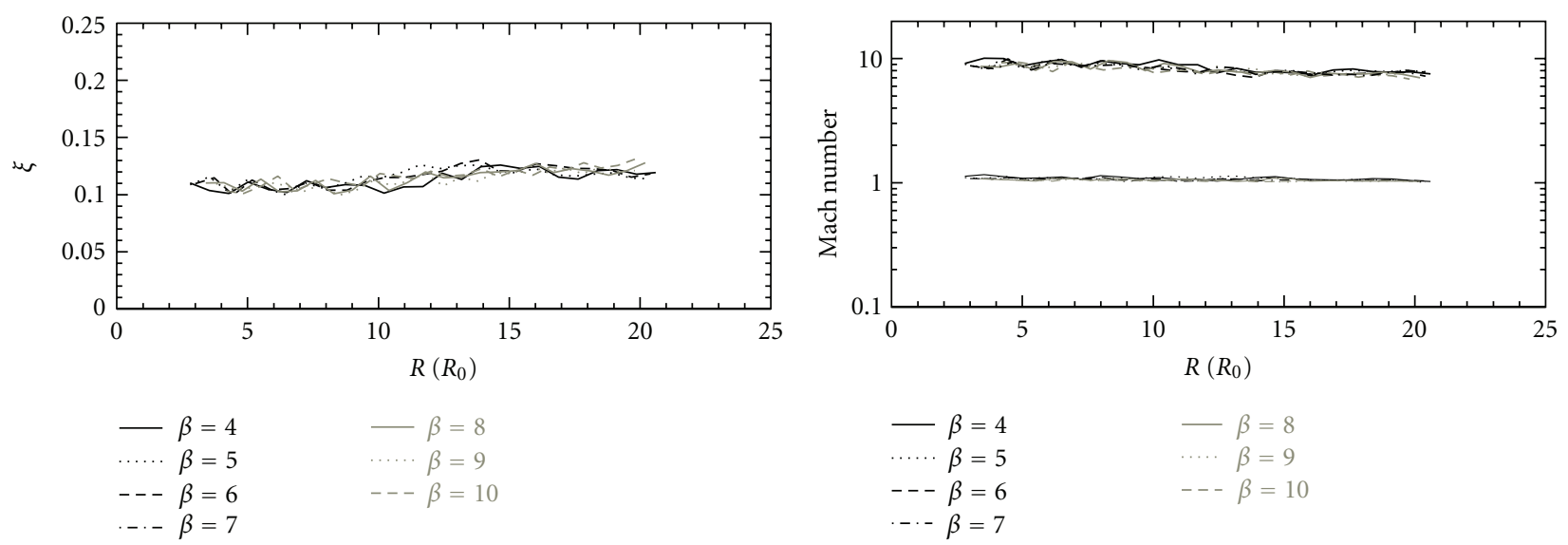

(a)
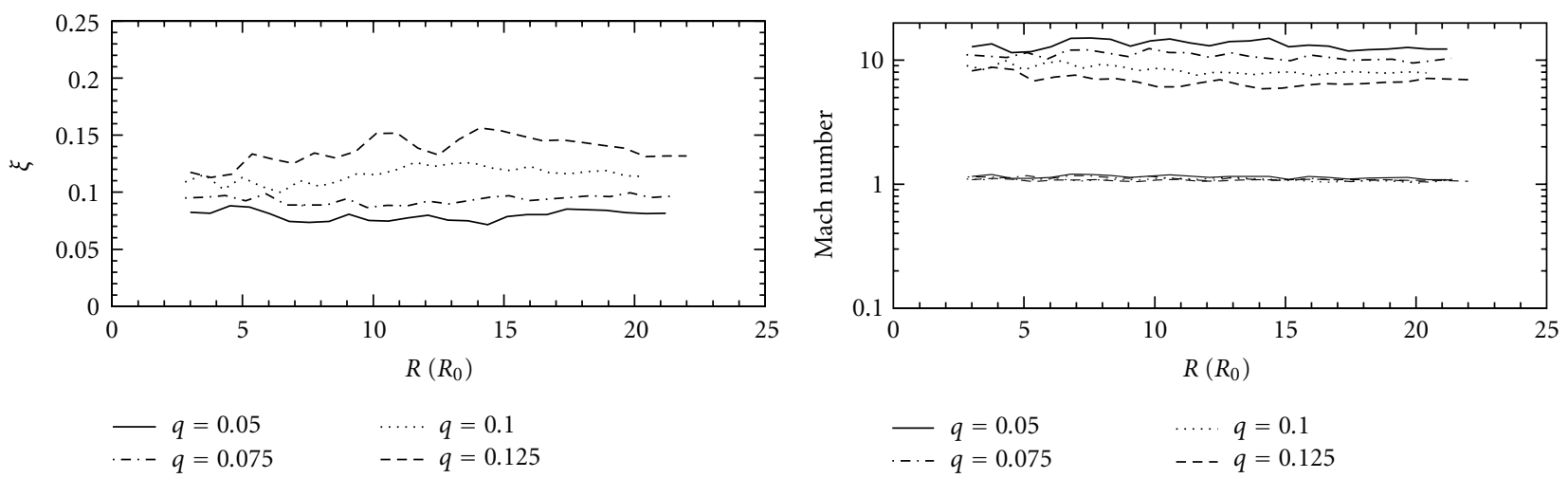

(c)

(d)

Figure 4: Nonlocal transport parameter $\xi(\mathrm{a}, \mathrm{c})$ and Mach Numbers (b, d) for simulations at various values of mass ratio $M_{\mathrm{disc}} / M$ and cooing parameter $\beta$, where $\beta$ varies, $M_{\text {disc }} / M=0.1$ and where $M_{\text {disc }} / M$ varies, $\beta=5$. For the Mach number plots, heavy lines denote the Mach number as measured in an inertial (static) frame, whereas light lines show the Doppler-shifted Mach number (measured in a frame corotating with the fluid). Taken from Cossins et al. [13].

result is invariant with either cooling rate or mass ratio. The gravitational instability therefore self-regulates so that not only is $Q \approx 1$, but also $\mathcal{M} \approx 1$. This result is intuitively reasonable in a quasisteady disc — subsonic waves would not impart any net heat to the disc (any compression heating is balanced by the corresponding rarefaction cooling), whereas a strong shock would be highly dissipative, leading to rapid evolution. Furthermore, the only way a fluid element can remain on a circular orbit when passing through an oblique (spiral) shock is if that shock wave has a unit Mach number.

Actually, we can also show that the above-mentioned dependence of the factor $\xi$ on the disc-to-star mass ratio $M_{\text {disc }} / M$ can be easily understood from the condition that these waves dissipate where they are almost sonic. In fact, using this sonic condition, we can rewrite (10) as

$$
\xi=\left|\frac{\Omega-\Omega_{p}}{\Omega}\right| \approx \frac{c_{s}}{v_{\phi}}=\frac{H}{R} \approx \frac{M_{\mathrm{disc}}}{M_{\star}},
$$

where $v_{\phi}=\Omega R$ is the azimuthal velocity of the disc, and the last equality holds for marginally stable discs $(Q \approx 1)$. This trend can actually be seen in Figure 4(c).
Thus the assumption of a local, viscous-like process for the transport associated with gravitational instabilities is only valid for light discs, where $M_{\mathrm{disc}} / M \ll 1$. It is in such cases that one can describe the secular evolution of the disc and the associated angular momentum transport in terms of an effective viscosity, and one can even choose to measure the stress induced by the spiral structure in units of the local pressure, thus obtaining an effective $\alpha_{\mathrm{sg}}$ value associated with gravitational instabilities. So, how large is the gravitationally induced $\alpha_{\mathrm{sg}}$ ? Lin and Pringle [6] propose the following parameterization:

$$
\alpha_{\mathrm{sg}}= \begin{cases}\eta\left(\frac{\bar{Q}^{2}}{Q^{2}}-1\right), & Q<\bar{Q}, \\ 0, & Q>\bar{Q} .\end{cases}
$$

Here $\bar{Q}$ is the value of $Q$ at which the disc becomes unstable to nonaxisymmetric perturbations and $\eta$ is a parameter to measure the strength of the induced torques. The above formulation is useful in practical cases, for example, when one wants to incorporate in a simple way the selfregulation mechanism in simple time-dependent models of 
self-gravitating discs. However, it lacks one important feature elucidated from the numerical simulations described above. In this picture, $\alpha_{s g}$ only depends on the local value of $Q$ and not on the cooling timescale $t_{\text {cool }}$, which we have seen controls so efficiently the development of the instability. In particular, for self-regulated discs, we expect $Q \approx \bar{Q}$ and the formula above would then produce a negligibly small $\alpha_{\mathrm{sg}}$, while we know that a finite amplitude spiral structure is present in self-regulated discs and indeed it is this spiral structure that provides the heating to balance the imposed cooling rate. On the other hand, we know that the process of self-regulation and the saturation of the gravitational perturbation is fundamentally related to thermal equilibrium in the disc: the saturation amplitude of the instability is such that the power dissipated through shocks in the disc is just enough to balance the imposed cooling (hence the inverse relation described above and displayed in Figure 2). In thermal equilibrium, the value of the viscosity parameter is simply related to the cooling rate [52]:

$$
\alpha_{\mathrm{sg}}=\left|\frac{\mathrm{d} \ln \Omega}{\mathrm{d} \ln R}\right|^{-2} \frac{1}{\gamma(\gamma-1) \Omega t_{\mathrm{cool}}}=\frac{4}{9 \gamma(\gamma-1)} \frac{1}{\beta},
$$

where the last equality holds in the case of a Keplerian disc. Indeed, the value of the stress induced by gravitational perturbation as computed directly from simulations of selfregulated discs $[21,23]$ agrees very well with the value predicted by (13). Thus, in a self-regulated state, not only the fractional amplitude of the density perturbations, but also the induced stress are inversely proportional to the cooling time. Indeed, one can also interpret the fragmentation threshold in terms of $\alpha_{\mathrm{sg}}$ rather than in terms of $\beta$ : there is a maximum value of the stress that can be supported by the disc without fragmenting [24]. Evaluating this critical $\alpha_{c}$ from (13) using the critical value of $\beta$, one finds that $\alpha_{c} \approx 0.05-0.1$.

Clearly, all this applies in cases where thermal equilibrium is simply established by a balance between the viscous heating and the radiative cooling. In many interesting cases (including the outskirts of AGN discs), irradiation from the central object is going to play a major role in determining the thermal balance. In such cases, (13) should be modified, and an interesting and only rarely discussed issue is what determines fragmentation: is it the stress exceeding the critical value $\alpha_{c}$, or is it the cooling time dropping below the critical value [53]?

What happens then for the cases where the disc mass is not much smaller than the central object mass? Here, we already know that we should expect deviations from the analysis discussed above, as transport should become significantly nonlocal. Once again, a change in behaviour has been observed in simulations [25]. The stress computed form the simulations does not agree anymore with (13), exceeding its prediction and peaking at values around unity. Furthermore, in these cases we have a situation where neither self-regulation nor fragmentation occurs. The disc simply cannot find a quasistationary nonlinear saturated, state and it keeps oscillating between periods of high spiral activity, where the stress would correspond to a local $\alpha$ of order unity, to periods of low activity, characterized by a temporarily high value of $Q$.

A summary of the various possible behaviours of a selfgravitating disc as a function of the three main dimensionless parameters is displayed graphically in Figure 5. Such picture summarizes effectively the various results discussed up to now.

Having discussed the main features of the gravitational instability in gaseous discs, I now turn to the application of the above results to the process that relates to the formation and growth of supermassive black holes in galactic nuclei.

\section{The Formation of Supermassive Black Hole Seeds}

One of the most important applications of the concepts described in the previous section to the context of supermassive black hole growth is the formation of massive $\mathrm{BH}$ seeds from direct gas collapse at high redshift.

This issue has become particularly important due to the recent discovery of active quasars up to redshift $z \sim 6$ [54, 55] and now even to a redshift as high as $z \sim 7$ [56], which indicates that supermassive black holes, with masses up to $10^{9} M_{\odot}$, were already in place when the Universe was only $10^{9}$ years old and beyond. This clearly requires that the black hole growth occurred at very high rates, with an average of $1 M_{\odot} /$ yr. Such a rapid early growth poses serious challenges to models of their formation.

Some models [57-59] assume that the seeds of supermassive black holes are the remnants of the zero-metallicity first stars (the so-called Population III stars), which are expected to be relatively massive $[60,61]$ and thus produce black holes with a mass of up to $100 M_{\odot}$. However, unless the efficiency of conversion of matter into energy through the accretion process is very low, it is impossible to grow the seeds to the required masses by $z \sim 6-7$ through Eddingtonlimited accretion [62]. The problem here is that when the accretion rate is large, the radiation pressure produced by the accretion luminosity can exceed the gravitational force of the black hole and thus exceeding the Eddington limit. Now, if the accretion efficiency $\epsilon=L / \dot{M} c^{2}$ exceeds $\approx 0.1$ (where $L$ is the accretion luminosity and $c$ is the speed of light), the Eddington limit does not allow the large accretion rates needed to grow the seeds fast enough to become bright AGN by $z \sim 6$ [62]. Note also that the Eddington limit is linearly proportional to the black hole mass, so that the problem of accreting at very high rates is particularly important in the earliest phases of the growth, when the black hole mass is small.

The efficiency is in turn dependent on the spin of the black hole, with high spin producing very large efficiencies $\epsilon \sim 0.5$. Accretion of matter naturally tends to spin up the hole [58] and hence to increase the efficiency, thus exceeding the Eddington limit for relatively low $\dot{M}$ and preventing a fast growth of the hole. While recent calculations $[63,64]$ show that it is possible to keep the hole spin low if the growth occurs through several small randomly oriented accretion episodes [65], we still have to face the issue of how to produce the high infall rates required. 


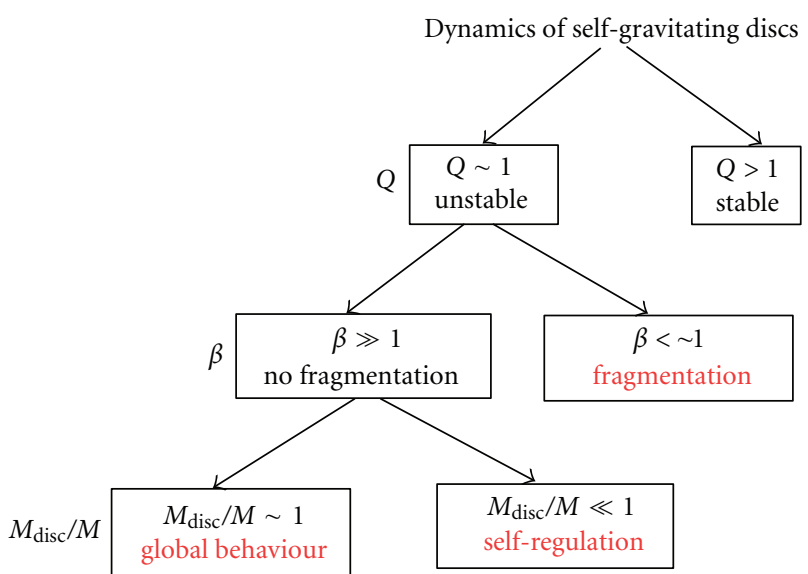

Figure 5: A simple diagram showing schematically the possible behaviour of a self-gravitating disc as a function of the three dimensionless parameters discussed here: $Q, \beta$, and $M_{\mathrm{disc}} / M$.

Alternative models propose the direct formation of more massive seeds with masses of about $10^{5} M_{\odot}$ directly out of the collapse of dense gas [66-72]. The key limiting factor for these models is the disposal of the angular momentum. Recently, it has been proposed [14, 71-73] that large-scale gravitational instabilities developing during the growth of pregalactic discs is the missing ingredient, able to funnel the required amount of gas into the center of the galaxy.

According to such models, the formation of the seeds of supermassive black holes occurs at a redshift $z \sim 10-15$, when the intergalactic medium had not been yet enriched by metals forming in the first stars. As a consequence, the chemical composition of the gas at this early epoch is essentially primordial, that is, the gas is mostly hydrogen and helium. The cooling properties of this gas are, therefore, relatively simple. In particular, in the absence of molecular hydrogen, the main coolant is provided by atomic hydrogen, for which the cooling timescale becomes extremely long for temperatures smaller than $\sim 10^{4} \mathrm{~K}$, and we thus expect the gas to reach thermal equilibrium at a temperature $T_{\text {gas }}$ of the order of $10^{4} \mathrm{~K}$.

Now, consider a dark matter halo (modeled, for simplicity, as a truncated singular isothermal sphere) of mass $M_{\text {halo }}$ and circular velocity $V_{h}$, extending out to $r_{h}=G M_{\text {halo }} / V_{h}^{2}$. We also assume that the halo contains a gas mass $M_{\text {gas }}=$ $m_{d} M_{\text {halo }}$, where $m_{d}$ is of the order of the universal baryonic fraction, $\approx 0.1$, whose angular momentum is $J_{\text {gas }}=j_{d} J$, where $j_{d} \sim m_{d}$. The angular momentum of the dark matter halo $J$ is expressed in terms of its spin parameter $\lambda=J|E|^{1 / 2} / G M_{\text {halo }}^{5 / 2}$, where $E$ is its total energy. The probability distribution of the spin parameter of dark matter halos can be obtained from cosmological $N$-body simulations in Warren et al. [74] and is well described by a log-normal distribution peaking at $\lambda=0.05$.

If the virial temperature of the halo $T_{\text {vir }} \propto V_{h}^{2}$ is larger than the gas temperature $T_{\text {gas }}$, the gas collapses and forms a rotationally supported disc, with circular velocity $V_{h}$, determined by the gravitational field of the halo. For low values of the spin parameter $\lambda$, the resulting disc can be compact and dense. In this case, during the infall of gas onto the disc, its density rises until the stability parameter $Q$ becomes of the order of unity. At this point, the disc starts developing a gravitational instability, which as we have seen above is able to efficiently redistribute angular momentum and allow accretion. Further infall of gas does not cause the density to rise much further, but rather it promotes an increasingly high accretion rate into the center. This process goes on until infall is over and the disc has attained a surface density low enough to be marginally gravitationally stable, that is, with $Q=\bar{Q}$. It is then possible to calculate what fraction of the infalling mass needs to be transported into the center to make the disc marginally stable, as a function of the main parameters involved. In this way, we get $[14,73]$

$$
M_{\mathrm{BH}}=m_{d} M_{\text {halo }}\left[1-\sqrt{\frac{8 \lambda}{m_{d} \overline{\mathrm{Q}}}\left(\frac{j_{d}}{m_{d}}\right)\left(\frac{T_{\mathrm{gas}}}{T_{\mathrm{vir}}}\right)^{1 / 2}}\right],
$$

where I have suggestively called $M_{\mathrm{BH}}$ the accreted mass, since this mass is the total mass available for the formation of the black hole seed in the center.

However, for large halo mass, the internal torques needed to redistribute the excess baryonic mass become too large to be sustained by the disc, which might then undergo fragmentation. We have seen in the previous sections that the maximum torque that can be delivered by a quasisteady selfregulated disc is of the order of $\alpha_{c} \approx 0.06$. Since the infall rate of gas from the halo is proportional to $T_{\text {vir }}^{3 / 2}$, we expect fragmentation when the virial temperature exceeds a critical value $T_{\max }$, given by (see [73] for details)

$$
\frac{T_{\max }}{T_{\text {gas }}}>\left(\frac{4 \alpha_{c}}{m_{d}} \frac{1}{1+M_{\mathrm{BH}} / m_{d} M_{\text {halo }}}\right)^{2 / 3} .
$$

Although it is possible, as mentioned above, that accretion proceeds even for larger values of $\alpha$ in a highly time-variable way when the disc mass is large, and it is also possible that accretion proceeds even in a fragmenting disc, we make here the conservative assumption that all halos that violate, (15), do fragment and do not accrete. Figure 6 illustrates the relationship between halo mass and black hole mass based on (14) for three different values of the spin parameter $\lambda$. The red line in Figure 6 corresponds to (15), so that halos on the right of the red line are expected to fragment. We can thus see that the typical mass fed into the center of such pregalactic disc is of the order of $10^{3} M_{\odot}$ up to $10^{5} M_{\odot}$. The typical accretion rates during this early epochs is of the order of $10^{-2} M_{\odot} / \mathrm{yr}$ [73]. If such high masses are assembled as seeds of supermassive black holes at redshift 10-15, it is then easy to grow through Eddington-limited accretion to $10^{9} M_{\odot}$ by $z=6$, as required by observations.

Equation (14) provides a powerful link between the properties of dark matter haloes and the mass of massive seed black holes that can grow within them. As shown, the amount of mass that will be concentrated in the central regions of these pregalactic discs depends only on halo properties (such as the spin parameter $\lambda$ and the fraction of baryonic mass that collapses to the disc $m_{d}$ ), on the ratio 


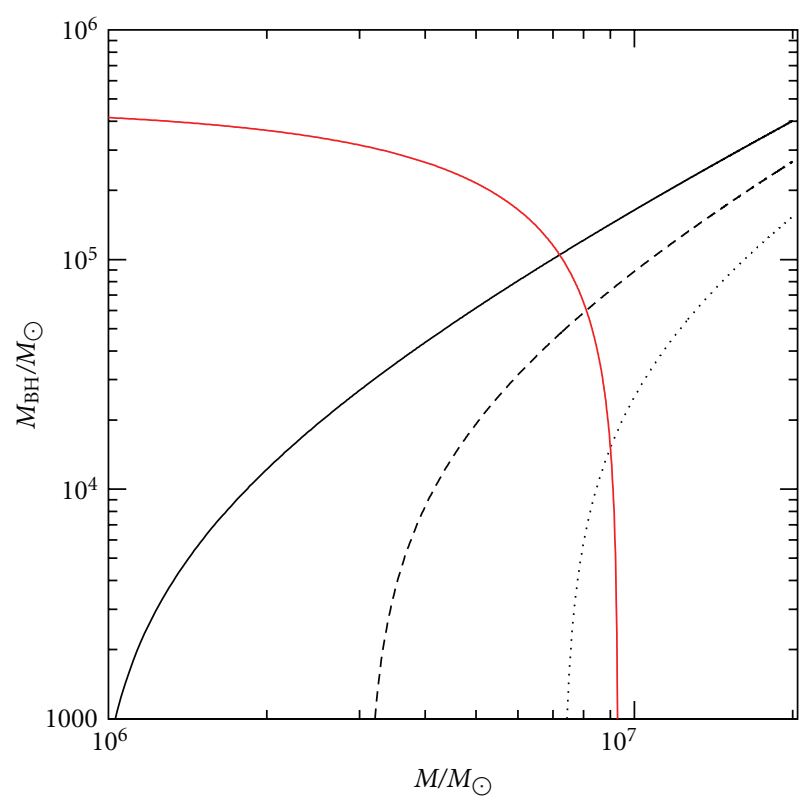

Figure 6: Mass available for the formation of the seed of a supermassive black hole in the center of pregalactic discs as a function of the mass of the parent dark matter halo (from [14]). The plots refer to the following choice of parameters: $\bar{Q}=2$, $T_{\text {gas }}=4000 \mathrm{~K}, m_{d}=j_{d}=0.05, \lambda=0.01$ (solid line), $\lambda=0.015$ (dashed line), and $\lambda=0.02$ (dotted line). The red curve shows the threshold for fragmentation from (15), with $\alpha_{c}=0.06$. Halos on the right of the red line give rise to fragmenting discs.

between gas temperature and halo virial temperature, and on the threshold value of $Q$, which has a very small range of variation around $\bar{Q} \approx 1$. This simple model has been used to calculate several properties of the black hole population at high redshift. In particular, from the distribution of halo masses and angular momentum, it is straightforward to derive the mass function of the supermassive black hole seeds Lodato and Natarajan [14], which turns out to be strongly peaked at around $10^{5} M_{\odot}$, as shown Figure $7(\mathrm{a})$. Furthermore, it is also possible to include such a simple prescription within evolutionary models that track the properties of the black hole population along cosmic time, such as merger tree models [15]. It is then interesting to see that the evolution of such a primordial seed population can naturally account for the current estimates of the density of black holes at low redshift (Figure 7(b)). In addition, an important and testable prediction of such models is that dwarf galaxies, which did not have any progenitor massive enough to seed a black hole, should not host a supermassive black hole. In particular, if the velocity dispersion of the galaxy is below $\sim 50 \mathrm{~km} / \mathrm{sec}$, the probability of hosting a black hole turns out to be negligibly small [15].

A key requirement for the above model to work is that the gas in the disc is cooling very inefficiently; otherwise, it would rapidly fragment and form stars rather than accreting to the center (see Section 2 above). Indeed, we require the gas to be free of the main coolants such as metals and molecular hydrogen. The process outlined above is thus going to be effective only before the intergalactic medium has been sufficiently enriched by metals and only where the gas is not excessively shielded by a UV background that tends to dissociate molecular hydrogen. This has led some to propose that the above mechanism only works at specific locations which satisfy the above conditions [75, 76]. Others [77] have instead proposed that even if fragmentation does occur, it would produce a compact stellar cluster whose eventual fate is still the formation of a supermassive black hole.

It is also interesting to mention that the process described analytically above has also been simulated numerically [78], and the results appear to be in substantial agreement with the analytical expectations.

Finally, note that the models described in this section only describe how can a substantial reservoir of mass be accumulated in the innermost regions of pregalactic discs. The eventual fate of this large amount of mass is not described here. Most probably, it will form a massive object at the center of the forming galaxy, such as a "quasistar" [79], where a seed black hole grows inside a large gaseous envelope which is accreting at rates which are super-Eddington for the hole, but not for the envelope itself. A detailed discussion of the physics associated with this is clearly beyond the scope of the present contribution.

\section{Gravitational Instabilities, Angular Momentum Transport, and Fragmentation in AGN Discs}

4.1. AGN Discs: Fragmenting or Nonfragmenting? The situation described above changes dramatically when we consider lower redshift, that is, if we now look at the outer disc in AGNs. As mentioned above, typically, the condition of marginal stability $Q \sim 1$ first occurs at a radius of the order of $0.01 \mathrm{pc}$ from the central black hole. One can easily calculate the cooling rate, and the associated cooling timescale, at this radius, to find that it is typically much smaller than the dynamical time [2, 39]. Stated otherwise, the heating rate needed to keep a marginally stable $(Q \sim 1)$ disc in thermal equilibrium is much larger than what can be provided by a viscous disc with reasonable values of $\alpha[1,80]$. If we now consult the results of the numerical simulations described in Section 2, we would simply conclude that the fate of such discs is to rapidly fragment into a number of bound objects. This is often interpreted as leading to intense star formation in the disc. However, it is worth noting that the dynamical time at $0.01-1 \mathrm{pc}$ from a $10^{8} M_{\odot}$ black hole (which is the time needed for the density perturbations to grow under the effect of the gravitational instability) (It is also easy to show that this is also the internal dynamical time of the fragments formed by instability in a $Q \sim 1$ disc.) is of the order of a few to a few thousand years. This is much shorter than the typical timescale associated with star formation in the solar neighbourhood, which is of the order of $10^{6}$ years. Now, clearly, star formation in the Galaxy occurs under significantly different conditions, as the local molecular clouds are much less dense than the fragments 


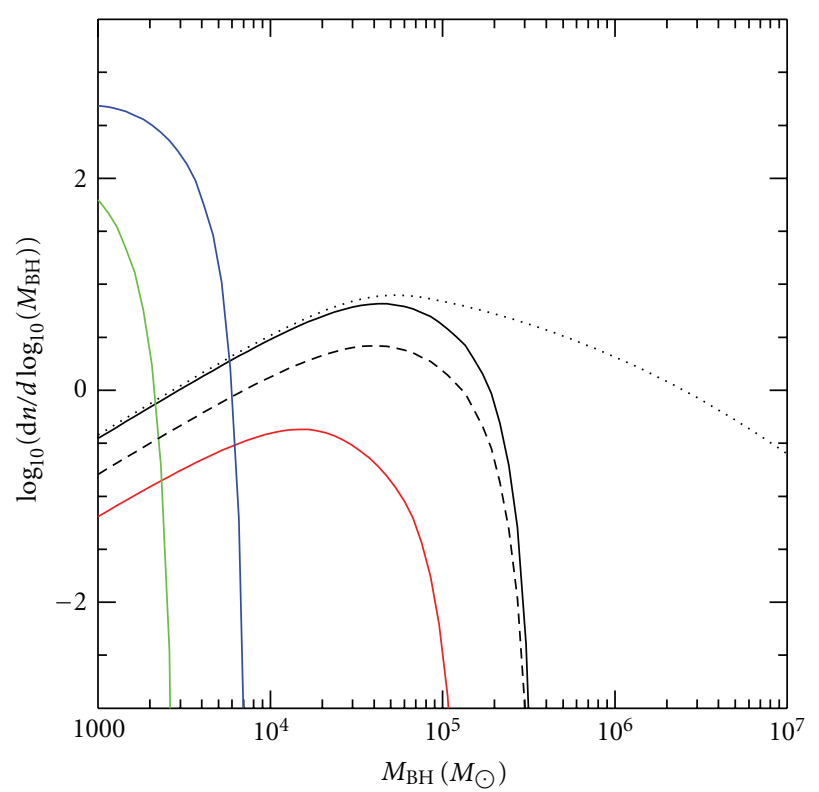

(a)

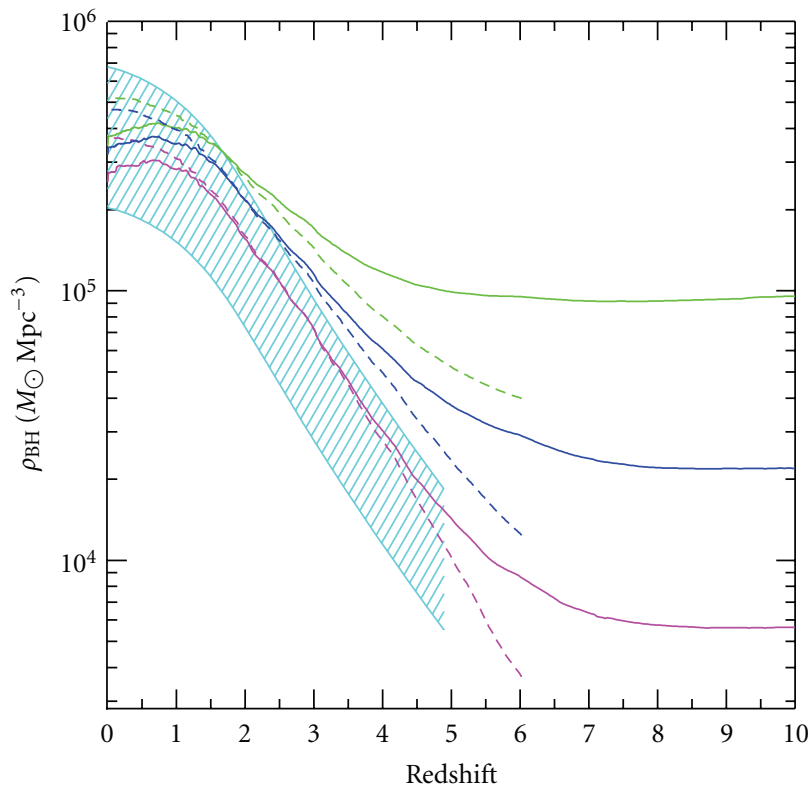

(b)

Figure 7: (a) Mass function of seed black holes predicted by the model based on (14) and (15). The black solid line refers to $z=10$, while the red line refers to $z=20$. The long-dashed line shows the effect of reducing $\bar{Q}$ from 2 to 1.5 . The short dashed line shows the effect of not including the possibility of fragmentation (more details can be found in [14]). (b) The integrated density of black holes predicted from a merger tree evolution of the black hole seed population. The three solid lines refer to three different choices of the parameters (see [15] for details). The dashed area indicates the observationally permitted region from estimates of the black hole density at low redshifts. At high redshift $(z \gtrsim 6)$, the density reflects the one attained after the seed formation phase, while the rise at lower redshifts indicates the growth through AGN activity.

produced in a fragmenting disc in this context. However, local star formation can also teach us something: indeed, the relatively long lifetime of molecular clouds in the Galaxy is due to the fact that rather than thermally supported, they are mostly supported by turbulent motions. The same might happen here: a fragmenting disc might produce a number of clouds whose dynamics is controlled by turbulent motions, which prevent their further collapse to form stars. In this case, the effective "cooling time," rather than the radiative timescale, would be the timescale for turbulence decay, which is of the order of the dynamical time and would thus be close to the threshold discussed in Section 2 [81].

A second thing to keep in mind is that if the disc extends to large distances, of the order of a parsec or so, its mass can become a significant fraction of the black hole mass and we might thus enter the regime where the dynamics associated with the gravitational instability is global rather than local. In this case, as already discussed, the energy balance should include some extra "global" terms, [51], arising from wave transport of energy, which might provide the required energy to prevent fragmentation in the outer disc. In this picture, a density wave might remove free rotational energy from the inner disc, but rather than dissipating it locally (as would a standard viscous process do), it might carry it a long way out along the wave and release it at large radii, where the wave is dissipated. As seen above, the evolution of such massive discs is generally highly variable, with episodes of strong accretion and black hole feeding followed by more quiescent periods where the accretion rate is small. Such a time variable accretion model has also been sometimes proposed by Collin and Zahn [82].

From the numerical point of view, simulations of the disc dynamics in this fragmenting regime are the most challenging, as the density in the clumps rapidly rises thus slowing down significantly the simulation. As a result, we still do not know in detail what is the fate of the disc: how much mass is turned into "stars" and what fraction of the disc mass is able to accrete to the central black hole rather than onto the forming stars [83]. Clearly, if most of the disc mass ends up in stars, it would be disastrous for black hole feeding, and we would thus conclude that only gas with very low angular momentum, which would circularize within the $Q \sim 1$ radius at $0.01 \mathrm{pc}$ would accrete onto the hole. This is the basic assumption behind the chaotic accretion scenario proposed recently $[84,85]$.

The presence of a significant stellar component within the disc can also in principle significantly affect the overall disc dynamics. Indeed, it has been proposed [86] that even if it constitutes a minor fraction of the overall disc mass, a stellar component in the disc is able to excite low- $m$ global spiral modes, even in a relatively low mass disc, and would thus provide a significant source of angular momentum transport, thus allowing accretion even from distances of order of several parsecs [19]. 
In the context delineated above, an important role is played by the evidence that has been gathered in the last few years, which points to the presence of a large number of young stars very close to the supermassive black hole at the center of our own Milky Way [87, 88]. In particular, most of these stars appear to belong to two distinct stellar discs orbiting at roughly the same distance to the black hole, that is, at a distance of $0.05-0.5 \mathrm{pc}[8,89]$. The most likely explanation for the origin of these stars in that they formed in situ and in particular from the fragmentation of a self-gravitating accretion disc $[89,90]$. Such observations thus fit naturally in the context described above, since we know that at parsec distances an AGN accretion disc would be self-gravitating and its cooling time is expected to be short enough to induce fragmentation. The conditions in the Galactic Center might be typical of other galaxies, where a nuclear starburst can be a result of the very same mechanism $[91,92]$.

4.2. Hints from Maser Dynamics. As mentioned above, there is clear observational evidence of the presence of significant mass in gas at parsec scales from the central black hole, in the form of maser emitting clumps. Such maser spots can effectively be used as a probe of the disc dynamics, as we can infer their rotation curve and hence probe the potential in the galactic nucleus. In most cases, as for example, the case of NGC 4258, the resulting rotation curve is very close to Keplerian [10], and it thus allows a very precise determination of the mass of the central $\mathrm{BH}$, which for NGC 4258 is $3.6 \times 10^{7} M_{\odot}$ (see also the recent compilation of Keplerian rotation curves obtained through maser emission by [93]).

However, in many other cases the rotation curve, while still displaying a smooth declining profile, as would be expected for a rotating disc, does not follow exactly Kepler's law. This is, for example, the case of NGC 1068 [11, 94], of the Circinus galaxy [95], and of NGC 3079 [96]. In particular, for the case of NGC 1068, the maser data are consistent with a circular velocity $v_{\phi} \propto r^{-0.31}$ [11]. Given the discussion above, which shows that at a scale of a fraction of a parsec, where the maser spots are detected, the disc can be self-gravitating, it is then tempting to attribute such (often small) deviation from Keplerian rotation to the contribution of the disc self-gravity.

A detailed fit to the circular velocity traced by water masers in NGC 1068 with a model which incorporates both the gravitational field of the black hole and that of the disc has been performed by Lodato and Bertin [33], by using self-regulated models of massive discs. The resulting black hole mass is $M=(8.0 \pm 0.3) 10^{6} M_{\odot}$ and the disc mass is approximately equal to the black hole mass. From the required disc surface density, it is then possible to obtain $\dot{M}=(28.1 \pm 0.2) \alpha M_{\odot} / \mathrm{yr}$. The mass accretion rate $\dot{M}$ can be estimated, for example, from the bolometric luminosity as $\dot{M} \approx 0.23 M_{\odot} / \mathrm{yr}$, and we thus obtain also an estimate of $\alpha \approx$ $8.3 \times 10^{-3}$, which is of the right order of magnitude as would be expected from the transport induced by gravitational instabilities.
4.3. Gravitational Instabilities and the Process of Binary Black Holes Merger. A related issue is connected to the process of black hole mergers. Black hole pairs are a natural byproduct of hierarchical galaxy formation, as a consequence of the merger of two galaxies each containing a nuclear black hole. Stellar dynamical processes are able to shrink the binary down to separations of the order of $1 \mathrm{pc}$ [97]. Additional gas dynamical processes can reduce the separation down to $0.1 \mathrm{pc}$ or so $[98,99]$. Below $0.001 \mathrm{pc}$, the emission of gravitational waves can shrink the binary further and lead to the merger of the two black holes. Such black hole mergers are indeed expected to be a primary source of gravitational radiation (and a prime target for gravitational wave detectors, such as LISA). However, an outstanding question is how to reduce the binary separation from $0.1 \mathrm{pc}$ to $0.001 \mathrm{pc}$. Given the essential lack of observational evidence for sub-pc black hole binaries, we know that the process needs to be fast. It has been frequently suggested that the role of gaseous discs at sub-pc scales can provide the necessary torques to produce such fast evolution.

The problem is in several ways connected with the issues discussed above. Indeed, both in the case of mass accretion to feed a single central black hole and in the case of reducing the separation of a black hole binary, the problem is how to dispose of the large orbital angular momentum. The internal torques within an accretion disc (whether "viscous" and thus local, or globally related to gravitational instabilities) could be the natural way to remove the excess angular momentum also in the case of a binary. In reality, in the case of a binary, the angular momentum transfer process is mediated by disc tides. A secondary black hole carves an annular gap within an accretion disc. It is the gravitational force between the disc and the satellite to remove the angular momentum from the satellite and reduce the binary separation. Viscous torques within the disc are then essential in redistributing the angular momentum taken up by the gas and transport it to large radii. The circumbinary disc then evolves subject to a source of angular momentum from its inner edge, in a way that has been termed a "decretion" disc [100]. The binary evolution timescale in this case is given by $[16,101,102]$

$$
t_{\text {shrink }}=\frac{M_{d}(a)+M_{s}}{M_{d}(a)} t_{v}
$$

where $t_{v}$ is the disc viscous timescale, $M_{\text {disc }}(a)=4 \pi \Sigma(a) a^{2}$ is a measure of the local disc mass at the binary separation $a$, and $M_{s}$ is the mass of the secondary black hole. The dependency on $t_{v}$ indicates the fact that viscous torques in the disc are ultimately responsible for the removal of angular momentum, while the factor depending on the relative mass of the disc and of the secondary indicates that if the inertia of the secondary black hole is much larger than the disc, then the shrinking must necessarily take much longer. At $1 \mathrm{pc}$, the viscous timescale is already of the order of $10^{8}$ years, and we thus see that if the disc mass is much less than the secondary mass, the shrinking timescale rapidly grows and can become exceedingly long for the merger to take place. Disc-assisted merger then requires large disc masses, comparable to the 


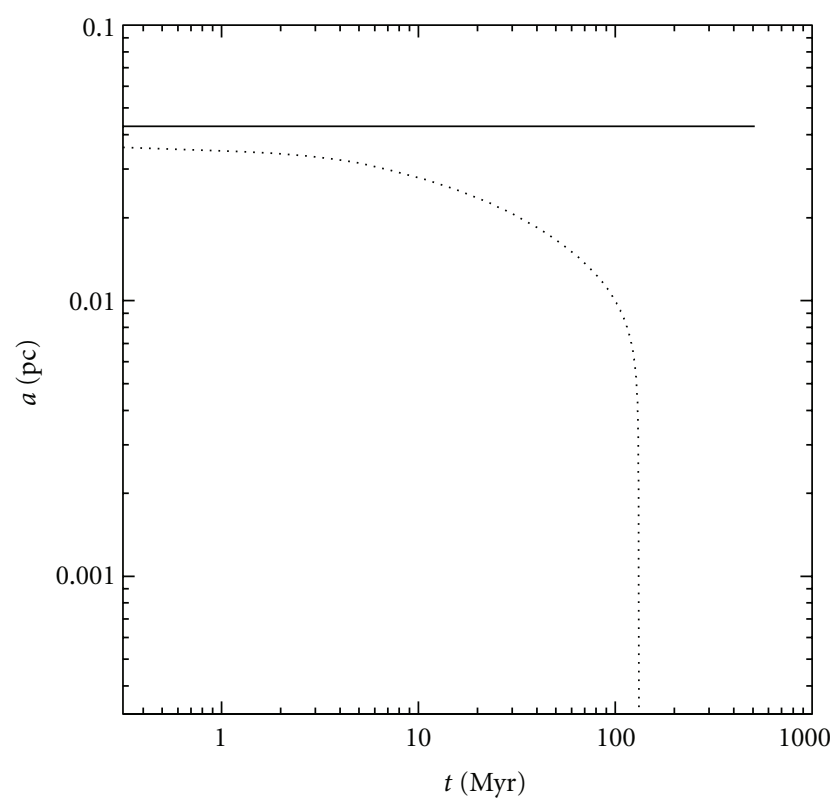

Figure 8: Evolution of the separation of a black hole binary with mass ratio $q=0.1$. The initial separation here was $a_{0}=0.05 \mathrm{pc}$ and the disc/secondary mass ratio is $M_{d} / M_{s}=1$. The solid curve shows the case where the disc is allowed to fragment and form stars, whereas the dotted line shows where star formation ignored. Star formation severely depletes the disc, and the remaining disc mass is not large enough to induce a black hole merger within a Hubble time. The figure taken from Lodato et al. [16].

secondary black hole, which are thus likely to be subject to gravitational instabilities.

Once again then, the dynamics of the gas disc at $\sim 0.01 \mathrm{pc}$ from the central black hole is essential in order to understand the evolution of the system. Lodato et al. [16] have studied the evolution of such systems. In particular, they have considered the case of a $10^{8} M_{\odot}$ primary black hole and of a secondary black hole with a mass ratio $q=0.1$. They have shown that, when neglecting the possible fragmentation associated with gravitational instabilities, disc torques are able to shrink the binary and allow the merger of the two black holes within a Hubble time. On the contrary, when the disc is subject to fragmentation in the self-gravitating regime (assuming that in the self-gravitating state, enough mass is turned into stars in order to keep the disc marginally stable), the shrinking stalls and the merger does not take place. One such calculations is shown in Figure 8. Here the initial separation of the binary was $0.05 \mathrm{pc}$ and the mass ratio was $q=0.1$. The two lines refer, respectively, to the case where the disc is allowed to fragment and form stars (solid line) and where fragmentation is ignored (dotted line). The effect of fragmentation is to inhibit completely the merger process.

Fragmentation thus has a severe impact on the ability of the disc to induce a black hole merger in a reasonable time. The rapid effect of fragmentation sets an upper limit to the effective mass of a gaseous disc. To avoid the disc becoming self-gravitating, the disc must have $Q \gtrsim 1$ and so must have a mass at most $M_{d} / M_{p} \lesssim H / R$ (see Section 2). On the other hand, we must also require that the disc mass be at least comparable to the secondary mass in order for the viscous torques to be able to remove the secondary angular momentum. With a typical aspect ratio $H / R$ of order of a few times $10^{-3}$, one can conclude that disc-assisted mergers only work for mass ratios $q \lesssim 0.001$.

\section{Conclusions}

AGN discs become self-gravitating at a distance of about $0.01 \mathrm{pc}$ from their central black hole. The development of gravitational instabilities in the disc can be both beneficial and detrimental for the process of black hole growth. Indeed, a beneficial effect is provided by the ability of gravitational instabilities to redistribute angular momentum within the disc and thus promote accretion. A detrimental effect is instead produced by the possibility of fragmentation, which could in principle turn most of the gas mass into star and thus remove it from the accretion flow. In this contribution, I have reviewed the recent progresses that we have made in the last ten years in our understanding of the nonlinear evolution of gravitational instabilities in gaseous discs, and in particular, on the parameters (most importantly, the disc cooling rate), that determine whether the instability saturates at a finite value-thus providing a quasisteady source of angular momentum transport in a self-regulated way-or rather fragments into bound objects.

Despite the impressive progresses made from the theoretical point of view, the application to AGN discs is not straightforward. Indeed, it turns out that the cooling timescale at the radius where the disc is self-gravitating is way to short to support a self-regulated state. This has led some authors to propose that AGN discs are effectively truncated at the self-gravitating radius. On the other hand, the evolution of a fragmenting disc is still not well understood, and it is not at all clear that the onset of fragmentation would totally preclude accretion. On the contrary, observational evidence such as the presence of Keplerian or quasi-Keplerian maser emitting gas at parsec scales in several AGNs, and the lack of observed black hole binaries at sub-pc scales-which in turn require the effective dynamical presence of a massive gaseous disc-hints to the importance of discs in the self-gravitating regime. Finally, it is worth noting that the presence of young stars in our own Galactic Center is indicative of the fact that star formation does effectively take place in the discs surrounding nuclear black holes.

A relatively better-understood evolution occurs at high redshifts, $z \sim 10$ or so, where the intergalactic medium was still not significantly polluted with metals and thus the cooling properties of the gas were significantly different. In these cases, fragmentation would be inhibited and the transport of angular momentum due to gravitational torques would naturally provide a way to accumulate large amounts of gas (up to $10^{5} M_{\odot}$ ) in the central regions of pregalactic discs, potentially opening the way to the rapid formation of black hole seeds by direct gas collapse. 


\section{References}

[1] G. Bertin and G. Lodato, "Thermal stability of self-gravitating, optically thin accretion disks," Astronomy and Astrophysics, vol. 370, no. 1, pp. 342-350, 2001.

[2] J. Goodman, "Self-gravity and quasi-stellar object discs," Monthly Notices of the Royal Astronomical Society, vol. 339, no. 4, pp. 937-948, 2003.

[3] P. I. Kolykhalov and R. A. Sunyaev, "Disk formation through accretion of stellar wind," Soviet Astronomy Letters, vol. 5, pp. 180-183, 1979.

[4] S. Collin and J. P. Zahn, "Star formation and evolution in accretion disks around massive black holes: star formation and evolution in accretion disks," Astronomy and Astrophysics, vol. 344, no. 2, pp. 433-449, 1999.

[5] D. N. C. Lin and J. E. Pringle, "A viscosity prescription for a self-gravitating accretion disc," Monthly Notices Royal Astronomical Society, vol. 225, pp. 607-613, 1987.

[6] D. N. C. Lin and J. E. Pringle, "The formation and initial evolution of protostellar disks," Astrophysical Journal, vol. 358, no. 2, pp. 515-524, 1990.

[7] I. Shlosman, J. Frank, and M. C. Begelman, "Bars within bars: a mechanism for fuelling active galactic nuclei," Nature, vol. 338, no. 6210, pp. 45-47, 1989.

[8] T. Paumard, R. Genzel, F. Martins et al., "The two young star disks in the central parsec of the galaxy: properties, dynamics, and formation," Astrophysical Journal, vol. 643, no. 2 I, article 033, pp. 1011-1035, 2006.

[9] R. Genzel, F. Eisenhauer, and S. Gillessen, "The Galactic center massive black hole and nuclear star cluster," Reviews of Modern Physics, vol. 82, no. 4, pp. 3121-3195, 2010.

[10] M. Miyoshi, J. Moran, J. Herrnstein et al., "Evidence for a black hole from high rotation velocities in a sub-parsec region of NGC4258," Nature, vol. 373, no. 6510, pp. 127-129, 1995.

[11] L. J. Greenhill, C. R. Gwinn, R. Antonucci, and R. Barvainis, "VLBI imaging of water maser emission from the nuclear torus of NGC 1068," Astrophysical Journal, vol. 472, no. 1, pp. L21-L24, 1996.

[12] P. T. Kondratko, L. J. Greenhill, and J. M. Moran, "Discovery of water maser emission in five AGNs and a possible correlation between water maser and nuclear 2-10 keV luminosities," Astrophysical Journal, vol. 652, no. 1 I, pp. 136145, 2006.

[13] P. Cossins, G. Lodato, and C. J. Clarke, "Characterizing the gravitational instability in cooling accretion discs," Monthly Notices of the Royal Astronomical Society, vol. 393, no. 4, pp. 1157-1173, 2009.

[14] G. Lodato and P. Natarajan, "The mass function of highredshift seed black holes," Monthly Notices of the Royal Astronomical Society, vol. 377, no. 1, pp. L64-L68, 2007.

[15] M. Volonteri, G. Lodato, and P. Natarajan, "The evolution of massive black hole seeds," Monthly Notices of the Royal Astronomical Society, vol. 383, no. 3, pp. 1079-1088, 2008.

[16] G. Lodato, S. Nayakshin, A. R. King, and J. E. Pringle, "Black hole mergers: can gas discs solve the "final parsec" problem?" Monthly Notices of the Royal Astronomical Society, vol. 398, no. 3, pp. 1392-1402, 2009.

[17] L. Ciotti and J. P. Ostriker, "Radiative feedback from massive black holes in elliptical galaxies: agn flaring and central starburst fueled by recycled gas," Astrophysical Journal, vol. 665, no. 2 I, pp. 1038-1056, 2007.

[18] L. Ciotti, J. P. Ostriker, and D. Proga, "Feedback from central black holes in elliptical galaxies. III. Models with both radiative and mechanical feedback," Astrophysical Journal, vol. 717, no. 2, pp. 708-723, 2010.

[19] P. F. Hopkins and E. Quataert, "How do massive black holes get their gas?" Monthly Notices of the Royal Astronomical Society, vol. 407, no. 3, pp. 1529-1564, 2010.

[20] C. F. Gammie, "Nonlinear outcome of gravitational instability in cooling, gaseous disks," Astrophysical Journal, vol. 553, no. 1, pp. 174-183, 2001.

[21] G. Lodato and W. K. M. Rice, "Testing the locality of transport in self-gravitating accretion discs," Monthly Notices of the Royal Astronomical Society, vol. 351, no. 2, pp. 630-642, 2004.

[22] A. C. Mejia, R. H. Durisen, M. K. Pickett, and K. Cai, "The thermal regulation of gravitational instabilities in protoplanetary disks. II. Extended simulations with varied cooling rates," Astrophysical Journal, vol. 619, no. 2 I, pp. 1098-1113, 2005.

[23] A. C. Boley, A. C. Mejía, R. H. Durisen, K. Cai, M. K. Pickett, and P. D'Alessio, “The thermal regulation of gravitational instabilities in protoplanetary disks. III. Simulations with radiative cooling and realistic opacities," Astrophysical Journal, vol. 651, no. 1 I, pp. 517-534, 2006.

[24] W. K.M. Rice, G. Lodato, and P. J. Armitage, "Investigating fragmentation conditions in self-gravitating accretion discs," Monthly Notices of the Royal Astronomical Society, vol. 364, no. 1, pp. L56-L60, 2005.

[25] G. Lodato and W. K. M. Rice, "Testing the locality of transport in self-gravitating accretion discs - II. The massive disc case," Monthly Notices of the Royal Astronomical Society, vol. 358, no. 4, pp. 1489-1500, 2005.

[26] G. Lodato, "Self-gravitating accretion discs," Nuovo Cimento Rivista Serie, vol. 30, no. 7, pp. 293-353, 2007.

[27] R. H. Durisen, A. P. Boss, L. Mayer, A. F. Nelson, T. Quinn, and W. K. M. Rice, "Gravitational instabilities in gaseous protoplanetary disks and implications for giant planet formation," in Protostars and Planets V, B. Reipurth, D. Jewitt, and K. Keil, Eds., pp. 607-622, University of Arizona Press, Tucson, Ariz, USA, 2007.

[28] G. Bertin and G. Lodato, "A class of self-gravitating accretion disks," Astronomy and Astrophysics, vol. 350, no. 2, pp. 694704, 1999.

[29] A. Toomre, "On the gravitational stability of a disk of stars," The Astrophysical Journal, vol. 139, p. 1217, 1964.

[30] G. Bertin, Dynamics of Galaxies, Cambridge University Press, Cambridge, UK, 2000.

[31] J. P. Ostriker and P. J. E. Peebles, "A Numerical study of the stability of flattened galaxies: or, can cold galaxies survive?" Astrophysical Journal, vol. 186, pp. 467-480, 1973.

[32] N. I. Shakura and R. A. Sunyaev, "Black holes in binary systems. Observational appearance," Astronomy \& Astrophysics, vol. 24, pp. 337-355, 1973.

[33] G. Lodato and G. Bertin, "Non-Keplerian rotation in the nucleus of NGC 1068: evidence for a massive accretion disk?" Astronomy and Astrophysics, vol. 398, no. 2, pp. 517-524, 2003.

[34] B. Paczyński, "A model of selfgravitating accretion disk with a hot corona," Acta Astronomica, vol. 28, no. 3, pp. 241-251, 1978.

[35] G. Bertin, "Self-regulated accretion disks," Astrophysical Journal, vol. 478, no. 2, pp. L71-L74, 1997.

[36] G. Laughlin and P. Bodenheimer, "Nonaxisymmetric evolution in protostellar disks," Astrophysical Journal, vol. 436, no. 1, pp. 335-354, 1994. 
[37] J. A. Sellwood and R. G. Carlberg, "Spiral instabilities provoked by accretion and star formation," Astrophysical Journal, vol. 282, pp. 61-74, 1984.

[38] L. Mayer, G. Lufkin, T. Quinn, and J. Wadsley, "Fragmentation of gravitationally unstable gaseous protoplanetary disks with radiative transfer," Astrophysical Journal, vol. 661, no. 1, pp. L77-L80, 2007.

[39] B. M. Johnson and C. F. Gammie, "Nonlinear outcome of gravitational instability in disks with realistic cooling," Astrophysical Journal, vol. 597, no. 1 I, pp. 131-141, 2003.

[40] P. Cossins, G. Lodato, and C. Clarke, "The effects of opacity on gravitational stability in protoplanetary discs," Monthly Notices of the Royal Astronomical Society, vol. 401, no. 4, pp. 2587-2598, 2010.

[41] M. J. Rees, "Opacity-limited hierarchical fragmentation and the masses of protostars," Monthly Notices of the Royal Astronomical Society, vol. 176, p. 483, 1976.

[42] J. Silk, "On the fragmentation of cosmic gas clouds. IIopacity-limited star formation," Astrophysical Journal, vol. 214, pp. 152-160, 1977.

[43] C. J. Clarke, E. Harper-Clark, and G. Lodato, "The response of self-gravitating protostellar discs to slow reduction in cooling time-scale: the fragmentation boundary revisited," Monthly Notices of the Royal Astronomical Society, vol. 381, no. 4, pp. 1543-1547, 2007.

[44] F. Meru and M. R. Bate, "Non-convergence of the critical cooling time-scale for fragmentation of self-gravitating discs," Monthly Notices of the Royal Astronomical Society, vol. 411, no. 1, pp. L1-L5, 2011.

[45] G. Lodato and C. J. Clarke, "Resolution requirements for smoothed particle hydrodynamics simulations of selfgravitating accretion discs," Monthly Notices of the Royal Astronomical Society, vol. 413, no. 4, pp. 2735-2740, 2011.

[46] G. Lodato and P. J. Cossins, "Smoothed Particle Hydrodynamics for astrophysical flows. The dynamics of protostellar discs," The European Physical Journal Plus, vol. 126, no. 4, 2011.

[47] S.-J. Paardekooper, C. Baruteau, and F. Meru, "Numerical convergence in self-gravitating disc simulations: initial conditions and edge effects," Monthly Notices of the Royal Astronomical Society, vol. 416, no. 1, pp. L65-L69, 2011.

[48] C. J. Clarke, "Pseudo-viscous modelling of self-gravitating discs and the formation of low mass ratio binaries," Monthly Notices of the Royal Astronomical Society, vol. 396, no. 2, pp. 1066-1074, 2009.

[49] R. R. Rafikov, "Properties of gravitoturbulent accretion disks," Astrophysical Journal, vol. 704, no. 1, pp. 281-291, 2009.

[50] D. Lynden-Bell and A. J. Kalnajs, "On the generating mechanism of spiral structure," Monthly Notices of the Royal Astronomical Society, vol. 157, p. 1, 1972.

[51] S. A. Balbus and J. C. B. Papaloizou, "On the dynamical foundations of $\alpha$ disks," Astrophysical Journal, vol. 521, no. 2, pp. 650-658, 1999.

[52] J. E. Pringle, "Accretion discs in astrophysics," Annual Review of Astronomy and Astrophysics, vol. 19, pp. 137-160, 1981.

[53] W. K. M. Rice, P. J. Armitage, G. Mamatsashvili, G. Lodato, and C. J. Clarke, "Stability of self-gravitating discs under irradiation," Monthly Notices of the Royal Astronomical Society, vol. 418, no. 2, pp. 1356-1362, 2011.

[54] X. Fan, J. F. Hennawi, G. T. Richards et al., "A survey of z > 5.7 quasars in the sloan digital sky survey. III. Discovery of five additional quasars," Astronomical Journal, vol. 128, no. 2, pp. 515-522, 2004.
[55] X. Fan, M. A. Strauss, G. T. Richards et al., "A survey of z > 5.7 quasars in the sloan digital sky survey. IV. Discovery of seven additional quasars," Astronomical Journal, vol. 131, no. 3, pp. 1203-1209, 2006.

[56] D. J. Mortlock, S. J. Warren, B. P. Venemans et al., "A luminous quasar at a redshift of $z=7.085$," Nature, vol. 474 , no. 7353, pp. 616-619, 2011.

[57] Z. Haiman and A. Loeb, "Observational signatures of the first quasars," Astrophysical Journal, vol. 503, no. 2, pp. 505-517, 1998.

[58] M. Volonteri, P. Madau, E. Quataert, and M. J. Rees, “The distribution and cosmic evolution of massive black hole spins," Astrophysical Journal, vol. 620, no. 1 I, pp. 69-77, 2005.

[59] J. S.B. Wyithe and A. Loeb, "Constraints on the process that regulates the growth of supermassive black holes based on the intrinsic scatter in the $\mathrm{M}_{b h-\sigma s p h}$ relation," Astrophysical Journal, vol. 634, pp. 910-920, 2005.

[60] T. Abel, G. L. Bryan, and M. L. Norman, "The formation and fragmentation of primordial molecular clouds," Astrophysical Journal, vol. 540, no. 1, pp. 39-44, 2000.

[61] V. Bromm, P. S. Coppi, and R. B. Larson, "The formation of the first stars. I. The primordial star-forming cloud," Astrophysical Journal, vol. 564, no. 1 I, pp. 23-51, 2002.

[62] M. Volonteri and M. J. Rees, "Rapid growth of high-redshift black holes," Astrophysical Journal, vol. 633, no. 2 I, pp. 624629, 2005.

[63] A. R. King, S. H. Lubow, G. I. Ogilvie, and J. E. Pringle, "Aligning spinning black holes and accretion discs," Monthly Notices of the Royal Astronomical Society, vol. 363, no. 1, pp. 49-56, 2005.

[64] G. Lodato and J. E. Pringle, "The evolution of misaligned accretion discs and spinning black holes," Monthly Notices of the Royal Astronomical Society, vol. 368, no. 3, pp. 1196-1208, 2006.

[65] A. R. King and J. E. Pringle, "Growing supermassive black holes by chaotic accretion," Monthly Notices of the Royal Astronomical Society, vol. 373, no. 1, pp. L90-L92, 2006.

[66] M. G. Haehnelt and M. J. Rees, "The formation of nuclei in newly formed galaxies and the evolution of the quasar population," Monthly Notices Royal Astronomical Society, vol. 263, no. 1, pp. 168-178, 1993.

[67] M. Umemura, A. Loeb, and E. L. Turner, "Early cosmic formation of massive black holes," Astrophysical Journal, vol. 419, no. 2, pp. 459-468, 1993.

[68] A. Loeb and F. A. Rasio, "Collapse of primordial gas clouds and the formation of quasar black holes," Astrophysical Journal, vol. 432, no. 1, pp. 52-61, 1994.

[69] D. J. Eisenstein and A. Loeb, "Origin of quasar progenitors from the collapse of low-spin cosmological perturbations," Astrophysical Journal, vol. 443, no. 1, pp. 11-17, 1995.

[70] V. Bromm and A. Loeb, "Formation of the first supermassive black holes," Astrophysical Journal, vol. 596, no. 1 I, pp. 3446, 2003.

[71] S. M. Koushiappas, J. S. Bullock, and A. Dekel, "Massive black hole seeds from low angular momentum material," Monthly Notices of the Royal Astronomical Society, vol. 354, no. 1, pp. 292-304, 2004.

[72] M. C. Begelman, M. Volonteri, and M. J. Rees, "Formation of supermassive black holes by direct collapse in pre-galactic haloes," Monthly Notices of the Royal Astronomical Society, vol. 370, no. 1, pp. 289-298, 2006.

[73] G. Lodato and P. Natarajan, "Supermassive black hole formation during the assembly of pre-galactic discs," Monthly 
Notices of the Royal Astronomical Society, vol. 371, no. 4, pp. 1813-1823, 2006.

[74] M. S. Warren, P. J. Quinn, J. K. Salmon, and W. H. Zurek, "Dark halos formed via dissipationless collapse. I. Shapes and alignment of angular momentum," Astrophysical Journal, vol. 399, no. 2, pp. 405-425, 1992.

[75] M. Dijkstra, Z. Haiman, A. Mesinger, and J. S. B. Wyithe, "Fluctuations in the high-redshift Lyman-Werner background: close halo pairs as the origin of supermassive black holes," Monthly Notices of the Royal Astronomical Society, vol. 391, no. 4, pp. 1961-1972, 2008.

[76] C. Shang, G. L. Bryan, and Z. Haiman, "Supermassive black hole formation by direct collapse: keeping protogalactic gas $\mathrm{H}_{2}$ free in dark matter haloes with virial temperatures $T_{\text {vir }}$ $>\operatorname{rsim} 10^{4} \mathrm{~K}$," Monthly Notices of the Royal Astronomical Society, vol. 402, no. 2, pp. 1249-1262, 2010.

[77] B. Devecchi and M. Volonteri, "Formation of the first nuclear clusters and massive black holes at high redshift," Astrophysical Journal, vol. 694, no. 1, pp. 302-313, 2009.

[78] L. Mayer, S. Kazantzidis, A. Escala, and S. Callegari, "Direct formation of supermassive black holes via multi-scale gas inflows in galaxy mergers," Nature, vol. 466, no. 7310, pp. 1082-1083, 2010.

[79] M. C. Begelman, E. M. Rossi, and P. J. Armitage, "Quasi-stars and the cosmic evolution of massive black holes," Monthly Notices of the Royal Astronomical Society, vol. 387, no. 4, pp. 1649-1659, 2008.

[80] E. Sirko and J. Goodman, "Spectral energy distributions of marginally self-gravitating quasi-stellar object discs," Monthly Notices of the Royal Astronomical Society, vol. 341, no. 2, pp. 501-508, 2003.

[81] M. C. Begelman and I. Shlosman, "Angular momentum transfer and lack of fragmentation in self-gravitating accretion flows," Astrophysical Journal, vol. 702, no. 1, pp. L5-L8, 2009.

[82] S. Collin and J. P. Zahn, "Star formation in accretion discs: from the Galactic center to active galactic nuclei," Astronomy and Astrophysics, vol. 477, no. 2, pp. 419-435, 2008.

[83] S. Nayakshin, J. Cuadra, and V. Springel, "Simulations of star formation in a gaseous disc around Sgr $\mathrm{A}^{*}$ - a failed active galactic nucleus," Monthly Notices of the Royal Astronomical Society, vol. 379, no. 1, pp. 21-33, 2007.

[84] A. R. King and J. E. Pringle, "Fuelling active galactic nuclei," Monthly Notices of the Royal Astronomical Society, vol. 377, no. 1, pp. L25-L28, 2007.

[85] A. R. King, J. E. Pringle, and J. A. Hofmann, "The evolution of black hole mass and spin in active galactic nuclei," Monthly Notices of the Royal Astronomical Society, vol. 385, no. 3, pp. 1621-1627, 2008.

[86] P. F. Hopkins and E. Quataert, "An analytic model of angular momentum transport by gravitational torques: from galaxies to massive black holes," Monthly Notices of the Royal Astronomical Society, vol. 415, no. 2, pp. 1027-1050, 2011.

[87] R. Genzel, R. Schödel, T. Ott et al., "The stellar cusp around the supermassive black hole in the Galactic center," Astrophysical Journal, vol. 594, no. 2 I, pp. 812-832, 2003.

[88] A. M. Ghez, S. Salim, S. D. Hornstein et al., "Stellar orbits around the galactic center black hole," Astrophysical Journal, vol. 620, no. 2 I, pp. 744-757, 2005.

[89] Y. Levin and A. M. Beloborodov, "Stellar disk in the Galactic center: a remnant of a dense accretion disk?" Astrophysical Journal, vol. 590, no. 1, pp. L33-L36, 2003.
[90] S. Nayakshin and R. Sunyaev, "The "missing" young stellar objects in the central parsec of the Galaxy: evidence for star formation in a massive accretion disc and a top-heavy initial mass function," Monthly Notices of the Royal Astronomical Society, vol. 364, no. 1, pp. L23-L27, 2005.

[91] S. Nayakshin, "Massive stars in subparsec rings around galactic centres," Monthly Notices of the Royal Astronomical Society, vol. 372, no. 1, pp. 143-150, 2006.

[92] Y. Levin, "Starbursts near supermassive black holes: young stars in the Galactic Centre, and gravitational waves in LISA band," Monthly Notices of the Royal Astronomical Society, vol. 374, no. 2, pp. 515-524, 2007.

[93] C. Y. Kuo, J. A. Braatz, J. J. Condon et al., "The megamaser cosmology project. III. Accurate masses of seven supermassive black holes in active galaxies with circumnuclear megamaser disks," Astrophysical Journal, vol. 727, no. 1, 2011.

[94] L. J. Greenhill and C. R. Gwinn, "VLBI imaging of water maser emission from a nuclear disk in NGC 1068," Astrophysics and Space Science, vol. 248, no. 1-2, pp. 261-267, 1997.

[95] L. J. Greenhill, P. T. Kondratko, J. E. J. Lovell et al., "The discovery of $\mathrm{H}_{2} \mathrm{O}$ maser emission in seven active galactic nuclei and at high velocities in the Circinus galaxy," Astrophysical Journal, vol. 582, no. 1, pp. L11-L14, 2003.

[96] P. T. Kondratko, L. J. Greenhill, and J. M. Moran, "Evidence for a geometrically thick self-gravitating accretion disk in NGC 3079," Astrophysical Journal, vol. 618, no. 2 I, pp. 618634, 2005.

[97] M. Milosavljević and D. Merritt, "Formation of galactic nuclei," Astrophysical Journal, vol. 563, no. 1, pp. 34-62, 2001.

[98] M. Dotti, M. Volonteri, A. Perego, M. Colpi, M. Ruszkowski, and F. Haardt, "Dual black holes in merger remnants - II. Spin evolution and gravitational recoil," Monthly Notices of the Royal Astronomical Society, vol. 402, no. 1, pp. 682-690, 2010.

[99] M. Dotti, M. Colpi, F. Haardt, and L. Mayer, "Supermassive black hole binaries in gaseous and stellar circumnuclear discs: orbital dynamics and gas accretion," Monthly Notices of the Royal Astronomical Society, vol. 379, no. 3, pp. 956-962, 2007.

[100] J. E. Pringle, "Self-induced warping of accretion discs," Monthly Notices of the Royal Astronomical Society, vol. 281, no. 1, pp. 357-361, 1996.

[101] D. Syer and C. J. Clarke, "Satellites in disks: regulating the accretion luminosity," Monthly Notices of the Royal Astronomical Society, vol. 277, p. 758, 1995.

[102] P. B. Ivanov, J. C. B. Papaloizou, and A. G. Polnarev, "The evolution of a supermassive binary caused by an accretion disc," Monthly Notices of the Royal Astronomical Society, vol. 307, no. 1, pp. 79-90, 1999. 

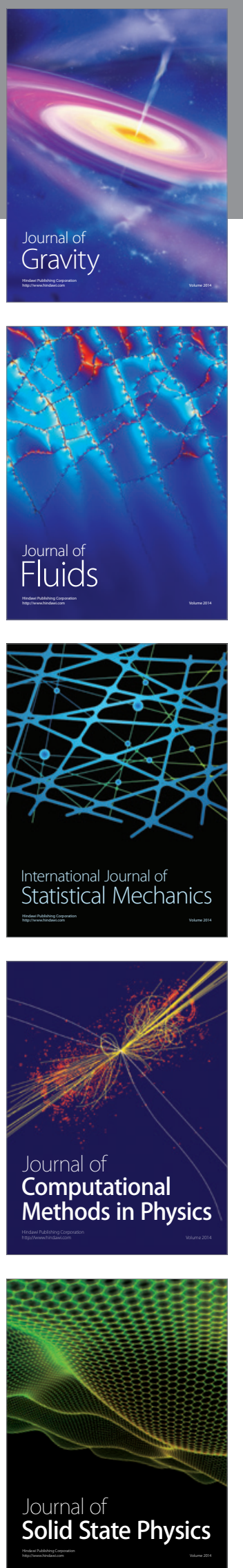

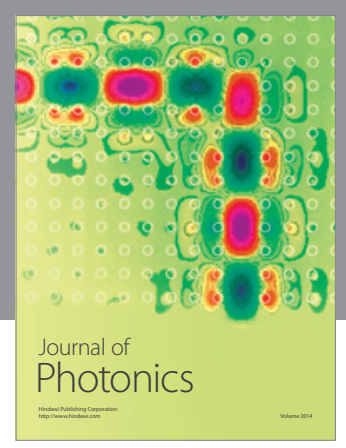

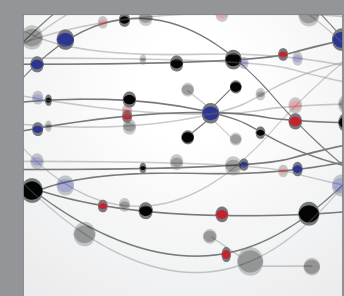

The Scientific World Journal
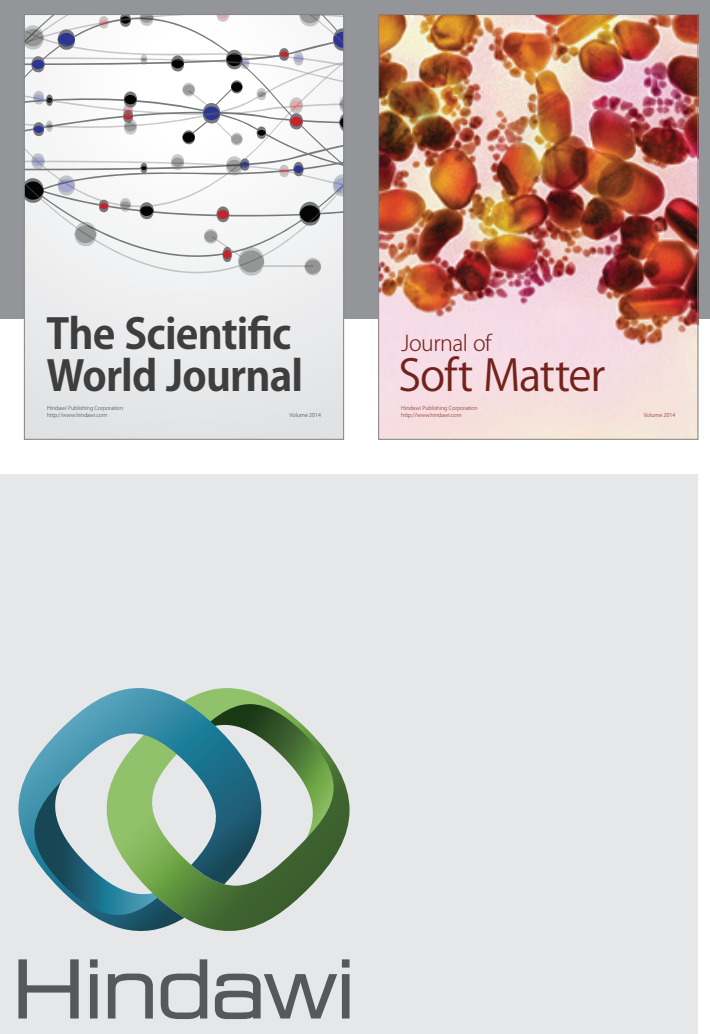

Submit your manuscripts at

http://www.hindawi.com
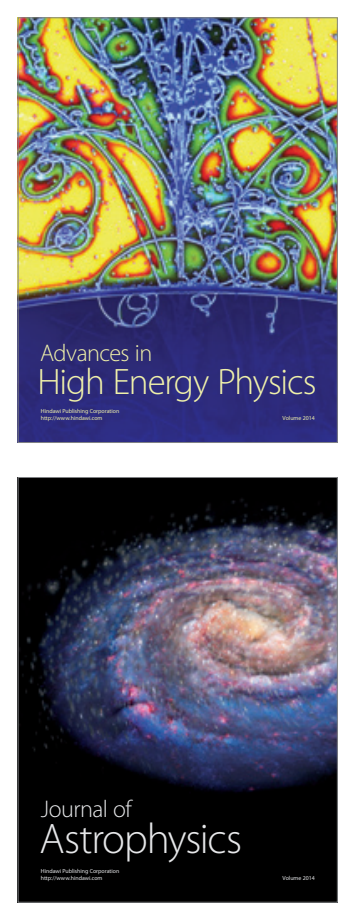
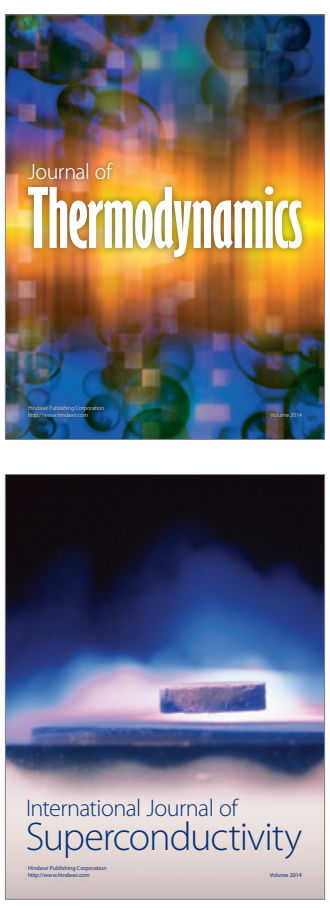
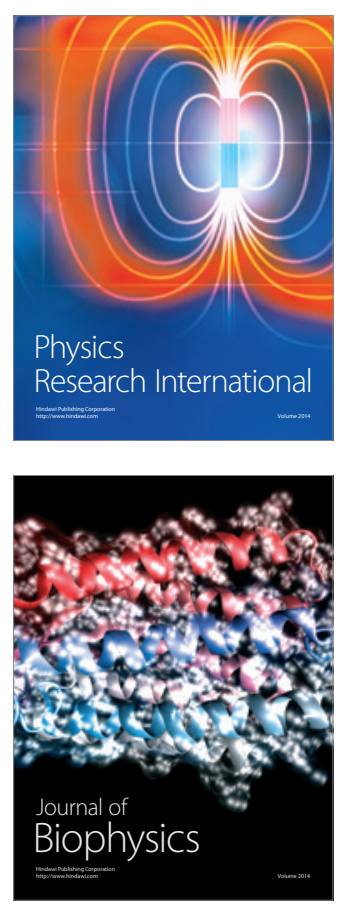
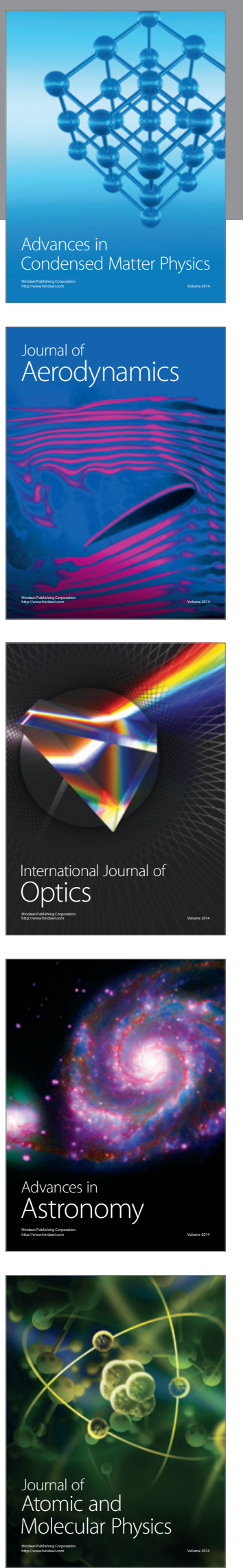